\title{
[1495] O sjorem magaaije! Fiktyf Joadsk etnolekt yn 'e Fryske literatuer
}

\author{
Bouke Slofstra
}

Abstract. In the past, Westerlauwers Friesland was already inhabited by a multilingual population, including speakers of local Frisian and nonFrisian vernaculars, the Dutch standard language and etnolects of foreign origin like those of German harvesters and Jewish merchants.

Frisian literature reflects this multilingual situation to some extent. The details of it have yet to be studied in a systematic way, however.

This case-study exemplifies how Jews were characterized in Frisian literature, especially drama. It turns out that the stereotypical Jewish character is presented as speaking a variety of artificial and real languages.

This study sheds some light on the question of how literature relates to reality, prejudice and language. It is argued that Frisian literature and multilingualism interconnect, the former existing in a multilingual reality, the latter being creatively manipulated by literary fiction.

\section{Ynlieding}

Meartaligens wie foar 1900, lykas no, in skaaimerk fan Fryslân en de Friezen sels. Dat feit wurdt wjerspegele yn de Fryske literatuer, en dat betsjut fansels folle mear as Fryske en Hollânske twataligens (Burger 1944, Meertens \& Wander 1958, Vries \& Breuker 1987, Jonkman \& Versloot 2008). By Feike van der Ploeg komme bygelyks sprekkers fan it Hylpersk foar. Yn it wurk fan de Halbertsma's oppenearje stedsjers en 'poepen' har yn har eigen taal, of alteast yn in karikatuer dêrfan. Waling Dykstra koe Biltsk en hat ek yn dy taal publisearre. Minder bekend is miskien it personaazje fan de Joadske keapman, dy't faak en folle yn 'e Fryske skriuwerij opfierd waard, meast yn toanielstikken.

De 'foddejoad' of 'lotterijjoad' yn 'e Fryske literatuer (en benammen op it toaniel) is hast altyd in typstra. Yn dy sin is der net sa folle ferskil mei oare flat characters op it toaniel (de gjirrige boer, de rabbelskûte ensafhuorthinne). Mar hy is ek yn 'e regel in bûtensteander, in oarenien om sa te sizzen. Op 'e planken wurdt dat oars-wêzen stal jûn meidat er in apart taaltsje praat. Dat taaltsje is in literêre keunsttaal. Dat smyt fuortendaliks in oantal fragen op. Hoe ferhâldt dy keunsttaal him ta de werklikheid? Is der

Us Wurk 69 (2020), s. 38-72; https://doi.org/10.21827/5d4811aa0744f 
in ûntjouwing yn dy keunsttaal? Is der in relaasje mei literêre sjenres of streamingen? Is der in relaasje mei foaroardielen fan de skriuwer sels? Feroaret it taaltsje as de stereotype Joad sels yn 'e rin fan 'e tiid feroaret (fan earme swalker ta rike jildliener)?

Ien bûnich antwurd op dy fragen is al earder jûn yn Van der Woude \& De Haan (1995:19):

Het stereotype beeld van de joden is nog het gemakkelijkst te vinden in de literatuur. In het Friese (en ook Nederlandse) volkstoneel zien we de joden vaak als karikatuur geportretteerd. De jood is dan vaak een niet helemaal eerlijke venter van textiel of loterijbriefjes die vee of waardevol antiek voor een (te) zacht prijsje wil opkopen en die zich bedient van een gebrekkig uitgesproken Nederlands of Stadsfries.

Hjirûnder sil ûndersocht wurde yn hoefier oft dat oardiel terjochte is. Dan sil ek in oare analyze (dy fan Brouwer 1931) oan 'e oarder komme.

Dit artikel is in tsjilling om in einfûgel te fangen. It sintrale tema yn dit stik is meartaligens yn de Fryske literatuer, mei it literêre Joadske etnolekt as case study. It soe nei myn betinken in goed ding wêze om mear yn it algemien út te sykjen hoe betrouber oft dy oare talen yn 'e Fryske literatuer werjûn wurde, en wêrom oft dat sa is. Mar men kin jin ek ôffreegje wat literêre skriuwers mei it gegeven fan dy meartaligens 'dogge'. Der is ommers net allinnich in meartalige kontekst om de Fryske skriuwerij hinne, mar meartaligens is ek in stik ark $y n$ de Fryske literatuer, en dat feit wurdt op ferskate wizen stal jûn. In skriuwer kin op ferskillende kreätive wizen mei it gegeven fan talen en taalkontakt 'boartsje', sa't hjirûnder sjen litten wurdt. Omdat sokke literêre meartaligens sa'n opfallende rol spilet yn de Fryske skriuwerij, soe it ferskynsel mear struktureel ûndersyk fertsjinje, nei myn betinken. Yn dit artikel wurdt dêr in smeet op dien. ${ }^{1}$

\section{Joaden yn Nederlân en yn it bysûnder yn Fryslân}

2.1 Skiednis

Joaden ha der sûnt de midsiuwen altyd yn 'e Lege Lannen west, oant it har troch Karel V en Philips II hast ûnmooglik makke waard om yn it Hillige Roomske Ryk te ferbliuwen. Om 1600 hinne is in nije stream fan Joaden yn Nederlân ynkommen, en om 1650 ek yn Fryslân (Brugmans \& Frank 1940, Blom e.o. 2017).

1. Mei in soad tank oan Eric Hoekstra, Alpita de Jong, André Looijenga en Abe de Vries. 
Yn Amsterdam wie in mienskip fan Portugeeske Joaden (dy't ek 'sefardim' neamd wurde) ûntstien. Hja hiene yn 'e regel maatskiplik sukses as heech oplate ambachtslju. Mar de measte Joaden yn Nederlân kamen út Dútslân wei. Dy Heechdútske Joaden (of 'asjkenazim') wurken faak as earme sutelers, bakkers of slachters.

Boargerrjochten krigen de Joaden yn Nederlân net earder as yn de Frânske tiid, op grûn fan it saneamde Decreet over den gelykstaat fan 1796. Dêrfoar wiene se feriene yn in frijwat autonome organisaasje, de saneamde Joodsche natie, mei in eigen rjochtspraak en ûnder lieding fan eigen gemeentelieders, dy't parnassim neamd waarden. It lidmaatskip fan in gild (sa't dat foar de Frânske tiid gebrûklik wie) stie foar Joaden net iepen.

Dy nije boargerlike status hat it yntegraasjeproses fan de Joaden, dat troch it hannelsferkear al oan 'e gong wie, befoardere. Heechdútske Joaden, dy't ûnderinoar wend wiene om Jiddysk te praten en te skriuwen, waarden geandewei better betroud mei de taal fan har omjouwing. Yn 'e rin fan 'e njoggentjinde iuw waard it Jiddysk foar hieltyd mear Joaden in sa goed as frjemde taal, dêr't allinnich wat wurden en siswizen fan oerbleaunen. Wy sille dat 'oerbliuwsel' mei de namme Joadsk Nederlânsk oantsjutte, fl. Sanders 2017b, Hinskens 2015, Van de Kamp \& Van der Wijk 2006.

De liberale Joadske elite, en letter de Nederlânske oerheid, hiene boppedat gjin ferlet fan it Jiddysk as ûnderwiistaal, dat it waard doe ek net op skoalle oanleard. De yntegraasje - en de dêrmei lykop rinnende emansipaasje - fan de Joaden yn 'e njoggentjinde iuw hat harren de wolfeart en it oansjen brocht dy't mannichien fan harren earder brek wienen. De tiid fan earmoed, efterstelling, swalkjen en problemen mei justysje rûn op syn ein (Brugmans \& Frank 1940, Weijtens 1971:35-6, Beem 1974, Schut 1995: 125-146, Lucassen \& Lucassen 2018).

Yn Fryslân tsjinne Ljouwert as in wichtich sintrum fan it Joadske libben yn 'e mediene, oanfolle troch oare mienskippen mei in eigen sjoel yn Harns, yn Boalsert, op 'e Gerdyk en op oare plakken. Om 1800 hinne naam it tal Joaden ta as gefolch fan arbeidsmigraasje út Hollân wei. De Ljouwerter mienskip of kille (sa'n 350 sielen yn 1800) hie op it hichtepunt yn 1870 sa'n 1800 leden. Dy synagogemienskip hie in behâldend karakter. Sadwaande bleau it Jiddysk dêr langer yn gebrûk as op oare plakken. De 'kroniken' fan de Ljouwerter Joadske mienskip, Sefer Zichronot neamd, waard oant it jier 1854 yn it Jiddysk byholden; dêrnei yn it Nederlânsk (Beem 1974: 164).

Dat nimt net wei dat in soad Joaden yn Fryslân sûnt de achttjinde iuw al twa- of meartalich wiene. Beem (ibidem) neamt it foarbyld fan in Adam Joseph Levy dy’t yn 1751 yn 'e Ljouwerter famyljerûnte seit: blief wat hier, 
ik hebbe jimme wat te seggen. Fan Joadske Fryskpraters yn 'e doarpen (Beem, ibidem) haw ik gjin bewizen fûn, mar it liket my net ûnwierskynlik ta. Famyljenammen lykas Drielsma ('Drylts-ma') en Terherne wize yn dy rjochting, sjoch bygelyks Beem 1953. ${ }^{2}$ Dat Nederlânske Joaden yn 'e njoggentjinde iuw ornaris de pleatslike streektaal yn 'e macht hiene, wurdt sawol yn 'e rieplachte literatuer (Meijer 1984, Groeneboer 1995, Broekema 2001) as yn literêre wurken (fan krekt nei 1900) dúdlik makke. Yn it wurk fan Sam Goudsmit (Kampen 1884-1954) wurdt Oeriselsk praat, bygelyks (fl. Goudsmit 1918). Benjamin Broekema (Warffum 1904-1942), ek fan Joadsk komôf, wie de skriuwer fan in grut tal toanielstikken yn Grinslânsk dialekt (Meertens \& Wander 1958, Meijer 1984 (ynlieding), Broekema 2001). It Joadske kameraatsje fan Merijntje Gijzen fan A.M. de Jong (1888-1943) praat heal-Brabânsk.

Oarsom ha ferskate Joadske wurden troch hannelskontakten foar 1900 in plak fûn yn it deistige taalgebrûk yn Nederlân (Moormann 2002; Den Besten 2006, 2010; Van de Kamp \& Van der Wijk 2006; Hinskens 2015). It Frysk wie gjin útsûndering: sjaggelje, goochem, poater, betûft, en, bygelyks, (op) skobberdebonk (libje) hawwe in Joadske etymology (Beem 1967, Tamminga 1961, WFT). ${ }^{3}$

\subsection{De Joad yn de skriuwerij yn Nederlân}

It klisjee-type fan de earme, swalkjende, net hielendal earlik hannel driuwende en opfallend krom pratende Heechdútske Joad as literêr personaazje is yn Nederlân om 1720 hinne ûntstien (Brugman \& Frank 1940, Alleman 1989). Yn 'e selde tiid kaam it leechlizzende wurd smous op: 'oorspr[onkelijk een] met name Hoogduitse Jood zonder vaste woon- of verblijfplaats en middelen van bestaan' (Van de Kamp \& Van der Wijk), dat letter in rûch skelwurd waard.

It wie net ûngebrûklik dat in skriuwer sa'n Joadsk personaazje "een soort half-Duits [liet] spreken dat voor Jiddisj wil doorgaan, en dat voor de gele-

2. De famyljenamme Leefsma (út: Levy) is in moai foarbyld fan oanpassing, krekt as it Frysk kostúm dat gauris troch Joadske froulju droegen waard (Beem 1974). Mar de nammen Drylsma en Terherne litte boppedat ek bekendheid mei de Fryske taal trochskimerje.

3. In soad wurden (sa as bajes en jatte) ha pas nei de Twadde Wrâldoarloch, fia it bargoens fan Amsterdam it Frysk berikt, sa't Tamminga (1961) ymplisyt oanjout (hy praat fan it Frysk fan "de jongerein”). Dy ûntjouwing hat der (fansels) ek yn it Nederlânsk west. Mar wurden as sjaggelje, op skobberdebonk en poater binne al earder, en út it streekrjochte kontakt mei Joadske keaplju, yn it Frysk bedarre. 
genheid [werd] vermengd met bepaalde stopwoorden als begotje, die ook later in de achttiende eeuw den joden nog bij voorkeur in de mond gelegd [werden]" (Brugmans \& Frank 1940 en fl. Alleman 136ff). By Nicolaas François Hoefnagel (Nederlandsche Overweeger, 1771) giet dat sa: maar hof je ets nieg mogte begreiffen ig eb ier een kykgas, taar kanje es altoemool hin sien, maar ig sol vort teis spregge. ${ }^{4}$ Dat skeelt in soad mei it Jiddysk dat yn werklikheid praat waard: Haynt iz purim, brider, / es iz der yon-tev groys. / Lomir zingen lider / un geyn fun hoyz tsu hoyz. ${ }^{5}$ (Resinte grammatika's fan it Jiddysk binne Jacobs 2005 en Birnbaum 2016.) Nei alle gedachten hat it ek de bedoeling fan Hoefnagel -en oaren-west om in sabeare heal-jiddysk, as kontakttaal, nei te bearen, want suver Jiddysk wie fansels foar yntern gebrûk ornearre.

Yn deselde tiid as by Hoefnagel waarden Joadske personaazjes 'sitearre' dy't in (heal fiktyf) soarte fan Joadsk Nederlânsk sprutsen, yn stee fan dat sabeare Jiddysk. ${ }^{6}$ In bekend foarbyld is de nijjierswinsk fan 'Mozes Polak en Soon' út itselde jier 1771 (of dêromhinne): Sjolem ('gegroet') hal degeen die deezen zillen zien, hof oore ('of horen') leezen. Sok fiktyf Joadsk Nederlânsk bleau net beheind ta de mindere of ûnbekende skriuwers, want it kaam ek foar by Multatuli (Woutertje Pietserse, Ideeën, 1862-1877) en Jacob van Lennep, De lotgevallen van Ferdinand Huyck (1840). Yn it lêstneamde wurk fine wy: 'Nha! al duwje me therug, dhaarom zelje toch ghezond blijven,' hernam de Jood, met de vasthoudenheid aan lieden van zijn beroep eigen: 'motje gheen halmenakkie 'ebben? gheen scharen, messen of photloodjens?' (fl. Van Oostendorp 2017).

De eigenskippen fan dit sabeare Joadsk Nederlânsk sille hjirûnder yn mear detail besprutsen wurde. Hoe't him dizze literêre keunsttaal ta de werklikheid ferhâlden hat, is net hielendal dúdlik, want it doedestiids besteande 'Joadsk Nederlânsk' hie sa'n lege status dat it hast allinnich as karikatuer op skrift kaam (mar ferlykje Winkler 1874, Hinskens 2015 en

4. Yn it Hollânsk fan Hoefnagel en syn lêzers: 'Maar als je het niet mocht begrijpen, dan heb ik hier een kijkgat (van een rarekiek), daar kun je het allemaal in zien; maar ik zal verder Duits spreken.'

5. 'Hjoed is it lottefeest, bruorren, / it is de grutte feestdei. / Lit ús lieten sjonge / en fan hûs ta hûs gean.'

6. Dokumintaasje oer 'Joadsk Nederlânsk' is te finen yn Van de Kamp \& Van der Wijk 2006. It besibbe 'Bargoens' wurdt besprutsen yn Moormann 1932-4, Den Besten (2006, 2010) en Endt \& Frerichs 1972. 'Bargoens' is in heterogene strjittetaal mei in soad Joadske wurden. 
Van de Kamp \& Van der Wijk 2006, hjirûnder). ${ }^{7}$ By skriuwers dy’t sels fan Joadsk komôf wiene, komme foarbylden fan dat 'etnolekt' wol foar (sjoch Van de Kamp \& Van der Wijk), mar folle minder en net sa karikaturaal. De ferneamde Joadske skriuwer Herman Heijermans lit in personaazje 'gewoan' Nederlânsk prate, fermingd mei in soad 'Joadske' wurden: Dat leeft zei Snoek na 'n stilte (...), dat leeft als een geschmadde jid ('gedoopte jood') - as die de krenke ('ziekte') het, denkt-ie eerst an z'n miese ('dood').

\section{Joadske personaazjes yn 'e Fryske literatuer}

\subsection{De midsiuwen en de iermoderne tiid: gjin personaazjes}

Yn 'e midsiuwen en de iermoderne tiid komt it wurd Joad wol foar, mar binne der noch gjin wurken fan literêre fiksje mei Joadske personaazjes.

It sizzen thet tha joden him thet lijf habbad binomen, yn Thet Freske Riim, ferwiist fansels nei it libben fan Kristus. Mar de Koadeks Aysma bringt ús op 'e hichte fan in bepaling út de eigen tiid: Dat xi is: jef en Jude queth, hy habbe en vrouwa, dar kersten ze, iefta joncfrowa, dat mey dat riucht nat thielda. ${ }^{9}$ ('De alfte útsûndering is: as in Joad seit dat er in frou hat dy't kristen is of jongfaam, dan mei it rjocht dat net duldzje'). Nei de midsiuwen is der yn ús boarnen gjin oanwizing foar Joadske oanwêzigens te finen oant de santjinde iuw, sa't te ferwachtsjen wie op grûn fan paragraaf 2.1. Wy sjogge yn it jier 1675 it sechje: Ofslytten biezems binne goed for de Joden. ${ }^{10}$ Gjin fan dizze sitaten jout in tige freonlik oardiel oer de Joaden. ${ }^{11}$ Net folle neutraler, mar wol gelearder, is in sitaat út in promoasjefers foar Ybe Fennema (Frjentsjer 1691): (...) in mei klem 't riucht mietyge gevielen / Ontrent de stoepen fin it Judsche Tjerk gewiel te toonen / dat de Jû̂d dit neat hie fin de Niel / Allyk Spencerus wol. ${ }^{12}$ ('En mei klam de rjochte fyzje oer de stûpen fan drokte yn de Joadske tsjerken oan te toanen, nammentlik

7. As yllustraasje hjirfan: Van Ginneken (1914) hie net begrepen dat it nijjiersfers fan 'Mozes Polak en Soon' in karikatuer is (fl. Van de Kamp \& Van der Wijk 2006:22).

8. 'De begrafenis', yn De gids, 1897. Sitearre by Van Ginneken (1914).

9. Codex Aysma, útjefte Buma e.o. 1993:327.

10. Ade Fries'ne sprekwirdden 1675, b181a (Taaldatabank Fryske Akademy).

11. Mear neutraal is it folgjende sitaat: Thit send tha fifine tekna ther er domes di koma skilun, ther sancte ieronimus fand escriuin an thera iothana bokon. ('Dit binne de fyftjin tekens dy't de hillige Hiëronymus yn 'e boeken fan de Joaden skreaun fûn.' Yn: Fyftjin tekens fan doemsdei). Mar dat giet oer (ûnderstelde) boeken fan lang ferlyn, net oer kontemporêne minsken.

12. Tiensma, Oon mijn wirde fruen (...), yn 1691 publisearre, nei oanlieding fan Ybe Fennema syn Frjentsjerter "disputatio theologica" oer "it wiere komôf fan de Hebriuwske riten”. Publisearre yn Feitsma 1956 (Estrikken nr xv, Grins 1956), s. 183. 
dat de Joad dit net út Egypte wei hie, sa’t John Spencer (1630-1693) ha wol.')

It boppesteande past yn it algemiene Nederlânske byld fan hoe't der yn dy tiid wol oer Joaden skreaun wurdt (en faak yn negative sin), mar dêr't de stereotype krompratende en sjacheljende 'smous' noch net as personaazje ferskynt (al soe men it sprekwurd út 1675 wol as in foarútwizing beskôgje kinne). Krekt foar 1800 feroaret dy sitewaasje yn de Fryske skriuwerij.

\subsection{Van der Ploeg en Meinerts: toanielwurk en sabeare Jiddysk}

Yn 'e toanielstikken Mayke Jakkeles (1777) en Jonge lieuws boosk (1778) fan de meniste dûmny Feike Hiddes van der Ploeg (1736-1790) spilet in eksplisyt Joadsk neamde figurant in rol. Mayke Jakkeles reizget te foet fan Hallum nei Ljouwert om de yntocht fan steedhâlder Willem V op 28 augustus 1777 by te wenjen. Underweis moetet se de Joadske sutelder David, dy't har by de stêdspoarte fan Ljouwert, yn alle drokte, twahûndert gûne ûntfytmannet.

Hik stae je goeje Margen te zeggen, jonge Vrauw! zil je reis naa de Stod ta? tan ebbe we kezelschip; hik pen een pittie ('beetje') te laat koomen, het Sjiep ('schip, Schiff') was wey. Hik kom, mottie wete, fan Hamelant (Ameland); hik swerf zo met Dozen en Mutzen door 't Lant. Hik wou wel geern formiddak ('vanmiddag') te Leeuwerten weze, want de Prins ('t is tog een braaf Man; Hij is ook Vrij-Eer van Hamelant,) zal fan te middag hingehaald worde, mit Aare Oogheid en Kyndere. Ei, ei, taar ebje een fraay koppeltje (nammentlik: dowen), tie pinne bi zokken hokkasie ('gelegenheid') nog hal een stuvertje waartig. Wat kosteze? Hik geefje for et stik een tubbeltje.

Yn it foarste plak falt it jin op dat David syn idiolekt hjir 'noch' in frijwat Dútsk, of better sein Jiddysk, lûd hat: kaufman, leben, geern, swaai ondert kilden ('twahûndert gûne'), sjoch Brugmans \& Frank 1940, Alleman (1989: 136ff), Groeneboer 1995. In oar skaaimerk fan (sabeare) Dútsk praat, yn persiflaazjefoarm, binne de stimleaze ploffers op plakken dêr't in stimhawwenden ferwachte wurdt: van te midag, tie pinne, waartig, tubbeltje, purger. In Dútsk pratende fjildwachter dy't yn deselde sêne foarkomt, docht itselde: das sol ein smous kedaan habbe.. Dyselde ferstimleazing wurdt letter ek troch de Halbertsma's brûkt as persiflaazje fan Dútskpraters ("doe pist ein 
Spitspoev"13). In lettere tiidgenoat fan de Halbertsma's, de Frânske skriuwer Guy de Maupassant (1850-1893), brûkt dy Dútske ferstimleazing yn 'e roman Boule de Suif: ("foulez-fous tescendre, messieurs et tames?"14). In foarbyld yn hjoeddeisk Frysk is it wurd poep ('Dútsker'), ôflaat fan Bube (WFT). Dêr soe in fonetyske eigenaardichheid mei anneks wêze kinne. Neffens guon fonetisy, sjoch ûnder oaren O'Brien \& Fagan (2016, paragraaf 6.4.1), ûnderskiede de Dútske 'lenis' ploffers $b, d, g$ har net fan $p, t, k$ troch in opposysje fan stimhawwend tsjinoer stimleas, oars as yn oare talen dêr't dat wol sa is (bygelyks Frânsk, Frysk, Nederlânsk), sadat Bube foar netDútskers mooglik op poep (e) liket.

David praat fierders in 'Joadsk' etnolekt mei in soad weilitten en tafoege $h$ 's (oogheid, Hameland), sa't hast alle sprekkers fan fiktyf Joadsk Nederlânsk hjir dwaan sille (fl. Van Ginneken 1914, Hinskens 2015, Van de Kamp \& Van der Wijk 2006).

Wy komme hjir by Van der Ploeg ek foar it earst de staan-te-konstruksje tsjin, dy't in skaaimerk is fan besteand en fiktyf Joadsk Nederlânsk (Hinskens 2015, Van de Kamp \& Van der Wijk 2006): Hik stae je goeje Margen te zeggen. ${ }^{15}$ Dy konstruksje soe nei alle gedachten in aspektuële betsjutting ha moatte, krekt as yn it Nederlânsk fan tsjintwurdich, mar dy ûntbrekt hjir yn 'e literêre keunsttaal. ${ }^{16}$

In oar skaaimerk fan de karikaturale Joad yn 'e literatuer is it feit dat er geregeldwei beswarrende formules brûkt, yn 'e measte gefallen om syn eigen earlikheid te beklamjen: Biggettje! ik sta een heerlijk Minsck te weezen. Biggettje is in njonkenfoarm fan begotje, dat hjirboppe al as karikaturaal Joadsk neamd waard (Brugman \& Frank 1940); it betsjut eins 'bij god' (WNT), en it is net as in spesifyk 'Joadsk' wurd opnommen yn Van de Kamp \& Van der Wijk (2006) of Beem (1967). Hik pin een Seun

13. De âld-militêr Ferdinant yn Minne Jorrits reis nei it Kollumer oproer seit "tat keloof ik" tsjin syn "puerfrau". It personaazje Toon út Detmold (Nordrhein-Westfalen) seit "doe pist ein Spitspoev" yn De reis nei de jichtmasters.

14. Lês: Voulez-vous descendre, messieurs et dames? 'Wolle jim útstappe, hearen en dames?'.

15. Sjoch de observaasje fan André Looijenga op 'e website Neerlandistiek (Van Oostendorp).

16. De staan-te-konstruksje hat yn it fiktive Joadske etnolekt heechút in 'progressive' betsjutting (d.w.s. it giet om in episoadyske, net-generike, hanneling). Yn 'e njoggentjinde iuw wie de aspektuële staan-te-konstruksje (ik sta hier de hele dag te werken) yn it Nederlânsk al bekend (Van der Horst). At dy in Joadske eftergrûn hat (fl. Van de Kamp \& Van der Wijk) is net dúdlik. Yn it Frysk kaam dy konstruksje ek al foar yn 'e njoggentjinde iuw (WFT sv stean). 
van David, py de Ouwe Lely Slois te Amsterdam; Rachel is mijn Mouder; 't pinne peide hopregte Israëls Purgers py main leben; ken je ze niet? Ze handele veul mit Kristyntje, over de Lange Piepe, giet David fierder. De begripen leven en gesondheid binne húsriem yn sokke beswarrende formules (bij mijn leven, gesondheid ensfh.), sa't hjirûnder ek te sjen is.

Foar in Amsterdamske Joad mei Ljouwerter konneksjes praat David in aardich mûltsjefol Frysk: naa de Staed ta, bi zokken hokkasie. ${ }^{17}$ Hy praat net of kwalik Stedsk (veul en seun soene Hollânsk wêze kinne, sjoch Van Bree \& Versloot (2008:115ff); (h)ingehaald is eksklusyf Nederlânsk). Ek yn lettere stikken praat it Joadske personaazje in skoft lang sa goed as gjin Stedsk, sa't wy hjirûnder sjen sille.

De literêre konvinsje liket foar te skriuwen dat Fryskpraters (yn dit gefal Mayke) en oarspraters (yn dit gefal David en de fjildwachter) altyd har eigen taal prate en altyd troch in oar te ferstean binne. Betizing is der allinnich - mei opsetsin en om it humoristyske effekt - as de fjildwachter Mayke freget út hokker "species" har twahûndert gûne bestie. Mayke antwurdet: Ik hie nen species, het wierne allegjerre Schillingen / zeis hondert en zeis en zextig / mey twa doebeltjes.

Yn Jonge lieuws boosk (1778), in oar stik fan Van der Ploeg, sjocht in Joseph ("ien smous") in bûsdoek op 'e grûn lizzen, dy't er oan 'e eigneresse sels ferkeapje wol (Hik sta pai mai leben forwonderd! Heen nieuwe zaktoek!). Hy freget (en krijt) swaay tibbeltjes ('twa dûbeltsjes') foar it finen. Dizze Joseph is in like earme swalker as de David fan Mayke Jakkeles: maar ebje nou niet een zoopje genever? Hik pin wurge; hik pin haleel fan Airlingen hafkelope; et empt is nat fan 't regene. Njonken de Joad Joseph komme ek twa Hylper fammen yn it stik foar, en dat soarget dúdlikernôch foar in komysk effekt. It "kromme praat komt no net fan Fryskpraters" (lykas faak it gefal wie yn de Frjentsjerter promoasjefersen) en "de clown is no gjin Fryske boer mar in heal-dútsk pratende joad" (Dykstra \& Oldenhof 1977:28). Miskien is dit kromme praat ferlykber mei wat Gubser (1998:139) en oaren 'Bühnen-Jiddisch' neame.

Yn It libben fan Aagtje IJsbrants (1779), dat oan Eelke Meinerts taskreaun is ${ }^{18}$, komt gjin Joadsk personaazje oan it wurd, mar Aagtje sels soe

17. Occasie, 'gelegenheid', wurdt by Van de Kamp \& Van der Wijk neamd as in 'Joadsk' wurd.

18. It stik is nei alle gedachten mei rjocht oan Meinerts taskreaun, sjoch Vries 1989. 
leafst alle wiken nije klean ha. Ik ken fen 't minste goed, dat nouw het uit dij mode komt, wol hette forkecepje, en oon 't ien of oor forruile; dij uitdraagsters en smouzen ken men alles oon kwijt wurde.

De 'smous' (yn dy perioade noch gjin rûch skelwurd) is yn dizze stikken in nuveraardige kreamer mei in sabeare Jiddysk aksint. By Van der Ploeg stelt er in oar syn jild (wat him by de Halbertsma's noch in kear foardwaan sil, mar dêrnei net mear). Joaden hiene, lykas earder sein, foar 1796 gjin boargerrjochten en mannichien fan harren hie fan klearebare earmoed te lijen. Maatskiplik hiene se in deistige èn offisjele status fan 'oare' minsken.

It niisneamde personaazje 'David' praat yn Mayke Jakkelis it sabeare Jiddysk fan syn fiktive tiidgenoat by Hoefnagel (1771), en 'noch' net it sabeare Joadsk Nederlânsk fan 'Mozes Polak en Soon' (ca 1770). Yn Ljouwert wie it Jiddysk doe noch yn libben gebrûk (sjoch 2.1). Mar miskien hat it iene net sa folle mei it oare te krijen, want it personaazje 'David' sels kaam út Hollân. Yn alle gefallen sil it sabeare Jiddysk nei Van der Ploeg út de Fryske literatuer weiwurde.

\subsection{De Halbertsma's: stereotypen en ynfoege $h$ 's}

By de Halbertsma's wurdt no en dan wat oer 'Jeuden' sein, en meastal as in algemiene útspraak en net oer yndividuële persoanen. ${ }^{19}$ De iennichste passaazje yn de Rimen en Teltsjes dêr't in Joadsk personaazje by de Halbertsma's as yndividu foarkomt is yn 'De âld kies fan Pîbomme' (Lapekoer, 1829), mar sels dêre hat de man gjin namme. It giet om in "keardel mei in swart bosk hier en in kromme noas" dy't in tafallige moeting hat mei Gabe Skroar, syn heit Pibe en 'master', as dy jûns te kuierjen binne op 'e terp fan Grou. Hy is it type fan de kiezzejoad of, ek wol, de kies-en-toskejoad (WFT): "Wel nha", sei de man, "bhen je mhal, boertje. Hik ben gehadmitteerd ${ }^{20}$ mhond-, khies- en tandmeester. Hik trekse uyt sonder pijn. (...) Daar misshe der drie". Nei in koart petear winskje de trije Grousters him in goede reis ta, wat ynhâldt dat hy blykber as in omswalkjende kiezzelûker syn brea fertsjinnet.

19. Wêrom oft de Halbertsma's it stedske wurd 'jeud' foar kar namen, is my net bekend. Blykber wie 'Joad' noch net it algemiene wurd yn it Frysk, want sels yn de Matteüsoersetting fan Joast giet it oer 'de jeuden'.

20. It wurd geadmitteerd betsjutte yn dy tiid net folle oars as 'bevoegd' (WNT), mar it kin wêze dat de kiezzelûker (foar 1796) syn fergunning út hannen fan de Joadske autoriteiten krigen hat (krekt as 'geadmitteerde slagers' en 'bakkers' doedestiids,, fl. Van de Kamp \& Van der Wijk sv, Beem 1974:64). 
Hjir praat de Joad net mear sabeare Jiddysk (lykas by Van der Ploeg) mar sabeare Joadsk Nederlânsk. En dat lêste is eigentlik mar skraachwurk it gefal. Mear as in hieltyd foar- en tuskenskode $h$ (krekt as by Van Lennep) hat it net te betsjutten, útsein de jimmer oanwêzige ynterjeksje na. Fan dy tuskenskode $h$ 's (bhen je mhal) is it folslein ûndúdlik wat se mei in histoarysk besteand Joadsk aksint te krijen ha moatte (Brugmans \& Frank 1940, Hinskens 2015, Van de Kamp \& Van der Wijk 2006, Sanders 2017a); it liket suver en klearebare literêr fiktyf 'Joadsk' te wêzen. Joast Halbertsma syn sizzen dat "de Joaden rûnom op 'e wrâld dyselde eigenskip [hawwe] dat se mei deselde net wurch te krijen aspiraasje dy't de taal fan har foarteam eigen wie, de talen fan alle gebieten dêr't se har ta wenjen set ha, beynfloedzje" $" 21$ (yn J.H. Halbertsma, Naoogst II, 1845, 396) liket elk bewiis te missen. Dy ynfoege $h$ liket sels in eksklusyf Nederlânsk skaaimerk fan sabeare Joadske spraak te wêzen. Yn Ut mine stromtid fan Fritz Reuter (1810-1874) komt it ommers net foar, mar yn de Nederlânske oersetting fan Gerrit Velderman wol. ${ }^{22}$ Dy nuveraardige ûnbekendheid mei wat paradoksaal lykje yn it gefal fan de taalkenner Joast Hiddes Halbertsma, mar dy is wol te ferklearjen. Halbertsma wie ynteressearre yn de Yndo-europeeske woartels fan syn eigen taal, net yn Semityske ynfloeden. ${ }^{23}$ Hy hat bygelyks mei gjin wurd skreaun oer de kolleezjes Easterske talen dy't er folge hie yn it Athenaeum yn Amsterdam (De Jong 2018: 34-5). (En hy hie, sa't liket, ek neilitten om by syn Joadske âld-rektor en taalkundige ynspirator Valentinus Slothouwer (1737-1822) nei te freegjen oft dy aspiraasje-teory wol doocht.)

Njonken de ûnwierskynlik brykpratende Joadske kiezzelûker komt yn itselde ferhaal in rillik oertsjûgjend Stedsk pratend personaazje foar: "Och hor i's, Pibe. Wij gain ook al zo feul met zukke dingen om, dat wij binne ook al halve dokters".

It type fan de earme swalkjende Joad makket him yn De reis nei de jichtmasters noch ien kear skuldich oan dieverij (ferlykje Aagtje IJsbrants fan Eelke Meinerts, hjirboppe): "Onder dy minsken wie in man, dy him yn

21. At in hoc Judaei ubique terrarum conveniunt, ut ipsa eadem aspiratione indefessa, quae linguae patrum propria erat, omnium regionum linguas, in quibus domicilium posuerunt, afficiant. (Werútjefte De Jong \& Slofstra 2017, 46-7).

22. Ich hab' mich einmal gemellt, es war in 'ner Sach' mit en Preußschen Kkannedaten, as ich hab' gemahnt den Kerl, hat er mir Brief geschrieben, ich soll nachlesen en Vers aus 'm christlichen Gesangbbuch. Dat wurdt yn 'e oersetting fan Velderman: Het was een zhaak met een phreisischen cannedaat; as ik heb ghemaant den vent heeft-i me geschreven een brief. Ik zou nhalezen een vers uit een chhristelijk gezhangboek.

23. De stúdzje fan de Jiddyske taal- en letterkunde hat in skoft lang te lijen hân fan dyselde fertutearzing by Dútske germanisten (Strauss \& Hoffmann). 
de merke in gouden haloosje fen in smous uut de boese troggelje litten hie. Dizze man klage jermlik hoe se him nou by de noos haan hiene." ${ }^{24}$ Dy stellerij sil hjirnei - better net te witten - net mear foarkomme yn 'e Fryske literatuer, mar de Joad sil noch wol regelmjittich in oar "by de noas" hawwe. ${ }^{25}$

In tiidgenoat fan de Halbertsma's, Klaas Cornelis Nijdam, skriuwt yn syn net-publisearre Botters-goed, in mijn liddige agenblikken (1828): Wat het dij oade smous in 't fet | Der stean ze raar om hinne. | Hij ropt en schrewt mar opgeset |Vif-doebbeld kin jim winne. Dit is it type fan de lûdroftige Joad op 'e merke, dat folle letter, by Simke Kloosterman, weromkomme sil.

\subsection{De Fryske 'folksskriuwerij' om Waling Dykstra hinne}

Yn dizze paragraaf wurdt earst it toanielwurk apart besprutsen, omdat dat it meast typearjende sjenre mei Joadske personaazjes is (fl. Brouwer 1931, Van der Woude \& De Haan 1995). Dêrnei komt it proazawurk oan 'e oarder (3.4.2). Yn 3.4.3 wurdt omtinken jûn oan de taal fan de Joadske personaazjes.

\subsubsection{Njoggentjinde-iuwsk reälisme: toanielstikken}

"[Onno Harmens] Sytstra, foarsitter fan de Krite yn Ljouwert, bitanke de sprekker en wiisde der noch op dat de joad by de Halbertsma's noch as ûnsympatyk ôfskildere waerd. Mar sûnt Waling Dykstra is der in kentering yn kommen", sa stiet te lêzen yn it kranteferslach fan Jelle Brouwer syn lêzing oer "de Joad yn de Fryske toanielstikken" (Ljouwerter Krante, 21 jannewaris 1931, tenei Brouwer 1931).

It stiet yndie net yn 'e kiif dat Waling Dykstra yn syn wurk hieltyd in ferljochte moraal trochskine liet. Dat sil hjirûnder ek bliken dwaan. Mar dat betsjut net dat hy hielendal frij wie fan negative oardielen oer Joaden. Yn syn Blommekranske for da Fryske berntsjes (s.j.) sketst er de folgjende utopy: In oengetiid soender bry | In preek dy ollemon haecht | In smous $d y$

24. De Halbertsma's jouwe mear yn it algemien in negatyf byld fan Joaden. Ferlykje: "lykas in jeud dy de boeren fermôgle figen fan "e kroade ferkeapet" (yn It Boalserter $N u t$ ) en "[o]p 'e jiermerke te Atherston krige hy rûzje mei in smous, dy him mei in pinmes yn de termen rippe woe; mar Abram koe mei it lytse mes net troch it fet rikke" (Grouster merke, 1839). De warskôging ûnder dat ferhaal (wachtsje jim foar brân en snijen; en benammen foar it besneine folk) wurdt yn in útjefte fan in jier letter (1840) wat mylder formulearre: in lit jim net snije, fen besneinen noch onbesneinen.

25. Ferlykje Alleman 1989 (par. 4.4) "de jood als schurk", Weijten 1971:36 en 38-41 en Schut 1995. 
nimmen bidraecht. Mar yn de toanielstikken fan Waling Dykstra en syn maten by útjouwerij Telenga (sjoch hjrûnder) komt wol in positiver byld nei foaren.

Waling Dykstra syn Op Maeijedei by Wigle Kwânseler (1891) is in hast letterlike toanielbewurking fan syn proazaferhaal De toalfte Maye fan 1851 (ferskynd yn 'e bondel Winterjounen by Gealeboer). De Joadske keapman en lommertsman Levie wit mei in liening tefoaren te kommen dat de sleauwe Wigle en syn húshâlden de wente ferlitte moatte. Hjir is Levie de 'eigentlike' kristenminske yn it selskip fan de sokses, de nepert en de rabbelskûte. Der is in soad kwea yn 'e wrâld, prakkeseart Wigle lûdop, mar tagelyk wirde der ek goede minsken foun - en ek wol goede Joaden. Dy deale $j a$, Levie hat my mar alderiwichste moai in doarn út 'e foet litsen hear. In oar motyf is dat fan de wrâldwize Joad en de sleauwe doarpeling (dy't hjir syn liende "bankje" fan fjirtich gûne by fersin yn in hoed benaaie lit). Levie sels slagget it om de boargemaster de ûnteigening fan de wente opkeare te litten: What! hik eb foor fheertich ghilde ghoed fan Whichle gekacht!" (...) Maer dat hik gekacht eb, is mijn eighedom, dhaer heeft de hijsheer ('huisheer') gheen recht op! In tredde motyf is it misbetrouwen foar de Joad oer (yn dit gefal by de frou fan de húshear). Dizze Levie liket op it type fan de 'edle Jude', nei it foarbyld fan Gotthold Ephraim Lessing syn roman Nathan der Weise út 1779 (fl. Brouwer 1931), dy’t ek yn de Hollânske literatuer wol foarkaam (Weijtens 1971: 41-43, Alleman 1989, Groeneboer 1995).

Yn Waling Dykstra syn fers In kristen en in joad (1904) wol in winkelman in earme frou gjin kredyt jaan. In joad, dy't om negoasje roun, hie 't hiele saekje heard en sjoen. Dit is ien fan de kearen dat de Joad de rol fan Thyl Ulespegel spilet. De snoade man dy't de minsken mei macht bedraacht foar it goede doel. Hy skewielt it frommis mei in dofke jild, dat de winkelman by fersin oannimt, mar efternei falsk blykt te wêzen. De ghrap is ghrof, maaar koopman, dat jou niet leper benne, spottet hy, yn heal 'Joadsk', heal Stedsk.

Yn syn fers In trien (1912) besjongt Waling Dykstra in Joadske keapman en lommertsman, "mei skealtsjes yn 'e hân", dy't begrutsjen hat mei in widdo dy't har ferstoarne man syn horloazje ferkeapje wol: Do sei er | ik wie siker mis, | 't is krekt as 't swierder wirden is. | Mar och, in widdou's trien weacht mear. Dat it Joadske personaazje net mear 'suver' fiktyf Joadsk Nederlânsk praat, is in skaaimerk fan de literatuer yn 'e tweintichste iuw, sa't wy hjirûnder yn paragrafen 3.5.1 en 3.5.2 sjen sille.

Klaas Aartsma (1838-1874) fan Berltsum wie muzyklearaar en lid fan it toanielselskip ‘Tot nut en genoegen' yn Menaam. Yn syn skriuwerij brûkte 
er sawol it Frysk as it Biltsk (It huushemelen), krekt as Waling Dykstra. It trije kear werprinte toanielstik It tsiende gebod of sa komme de slînders to pas (1862) waard útjûn by Telenga yn Frjentsjer (dêr't Waling-om yn tsjinst wie). "It is biarbeide nei in intrigue fen mîn frieun W. Dykstra", seit it foaropwurd. It giet eins om it besteande folksferhaal 'Vrijers in de kast' (Meertens, Volksverhalenbank): de Harnzer seeman Jelbe besiket de Joadske keapman Nathan in 'weardefolle' hingelmatte te ferkeapjen. Nathan hat gjin ynteresse: $O$ whaai! Houd je kapoeris ('brot'). Dhenkje dhat ik bhen komme staan gek te worden? Bhij me magere siel! Bij honse Haarsfader Jakob. Mar dan snijt Jelbe in tou los en de trije frijers fan syn frou Wikje falle op 'e grûn. Aartsma hat sûnder mis gebrûk makke fan Hollânske foarbylden, want it hjir folgjende lietsje komt foar in grut part streekrjocht út De klagende Jood fan 1852 (fl. Sanders 2017a): ${ }^{26}$

Nah khijk! hik dhoe jelui te hweten | Has dhat ik khoopman Nathan ben, | ' $k$ Khoop kleêren, houd hof half ferslethen, | Has hik mhaer moos (jild) ferdhiene ken. | Heeft soms de bhaes what houde broekhe, | Hof wel de frou in stikken jak, | Nah, sei kaan narr ['sei kein Narr, wês net gek'], wil 't mij ferkhoope, | Hik stop it halles hin e foddesak. | Kleêrkhoop! Kleêrkhoop!

Notaris Hermanus Alma (1839-1915) fan Menaam wie krekt as Klaas Aartsma in warber lid fan 'Tot nut en genoegen'. Hy skreau in stikmannich toanielstikken mei in ferljochte moraal, bygelyks Rabberij beskamme en woldwaen beleanne (1865) en Frijheid, lîkheid en broerskip (1866). Yn syn toanielstik Trye jieren letter of Levi Smoel de Lotteryjoad fan 1863, dat ek wer by Telenga útjûn waard, komt it motyf fan de lotterij oan 'e oarder. De lotterij wurdt hjir, lykas ornaris, as in tsjuster kwea beskôge, ferlykber mei drankmisbrûk en byleauwe. De Joad dy't lotterijbriefkes ferkeapet is yn sokke gefallen net folle mear as in ynstrumint foar it ferhaal, sjoch ek Brouwer 1931. ${ }^{27}$ Yn Alma syn stik is de boer Jan Troef de lotsjeferkeaper Levi Smoel fyftich gûne skuldich, en hy skammet him foar syn meidwaan oan de lotterij. Letter ferskynt "Levi" sels op it toaniel: Ghoeije navond, mhensc[h]e! Nah, hik sie 't mhe wel, je bhent whaarachtig verschrikt, that hik hier kom vhoor je te sthaan. Mhaar, ho sjorem magaaije! hhas jheliede

26. Anoniem, De Nederlandsche zanger in alle gezelschappen (1852), side 15. https:// www.dbnl.org/tekst/_ned022nede01_01/_ned022nede01_01_0007.php

27. In iere útsûndering op dy lottery-krityk is Tsien tuwsen uwt de lottery (1841) fan Harmen Sytstra, dêr't immen in winnend lot fan de "smous" kocht hat en út 'e skroeven is fan blidens. 
zelt hebbe khom staan te hwete, what boodschap hof hik, Levi Smoel, han jhullie heb, nah nah,!, je zhoudt mhe niet staande kijke has Piet Snot ('bedremmeld staan te kijken'). Jan hat ommers in priis fan 2000 gûne wûn, mar moat earst foar ûntfangst tekenje (heerst een bewhijs, has thatje het hontvange hebt). Dyselde "Levi" komt letter werom en lit - wannear't er net wolkom is - in dokumint sjen dat Jan in skuld fan twatûzen gûne hat by in kunde mei de namme Klaas. "Levi" en Klaas blike ien en deselde persoan te wêzen en it dokumint is datselde "bewhijs". Op it lêst komt alles yn oarder. It motyf fan it misbetrouwen is op "e nij oanwêzich ("it falt my net iens fan dy smous ôf dat er sa earlik is"). Boppedat wurdt de sabeare "Levi" troch de boerinne priizge om syn oertsjûgjende 'Joadske' etnolekt, dat - fansels - in suver literêr-fiktyf taaltsje is.

De boekdrukker Roel Kiemstra fan De Gerdyk (1830-1912) wie de auteur fan it stik 't Kjeallefel en it lotterybriefke (1862), dat trije kear werprinte waard. It is in smjunteroman yn toanielfoarm oer de slûkjende slachter Siebe en syn Joadske maat Mozes, dy't slûchslimmer blike te wêzen as it gesach yn de persoan fan twa kommizen, ien Hollânskprater en ien Stedsjer ("Heb je het huus alleenig of is jou man ook thuus?"). Mozes praat in karikaturale soarte fan Joadsk Nederlânsk. Dêrnjonken wit er, alwer, mear fan 'e wrâld as de minsken om him hinne. Oft er hook wat nieuws is! Hin Hitaalje, mot je wete, slaan ze mekaar bij honderden kapoet; hoveral oorlog, pest en pestilensie. Siebe is grif noait fierder kommen as de trein nei Ljouwert, seit er spotsk.

Nieske Stamsma. En forhael ût ûs tîd $(1869)^{28}$ is fan Marten Pieters Troelstra, in omke fan Piter Jelles. De Joad Levi is hjir in "earste skachler" ('sjachelder') dy't yn "ald gûd, alde klean, fudden en bunken" docht en regelmjittich nei Amsterdam reizget. Hy nimt by dy gelegenheid ek brieven fan in jongfeint (Pibe) foar syn faam (Nieske), en oarsom, mei. Mar Pibe syn rivaal Simen krijt Levi sa fier dat er him mei bedragerij en rabberspraat Pibe syn faam ûntfytmanje lit. "Nah", andere disse, "nah, dhie vhind ik haerdich! Whaarom sou ik niet whille?" De Joad jout allinnich om jild: "Gheld is de ziel van de negotie".

It blijspul In rôt yn 'e stap (1885) fan H.E. Bakker giet oer in ryk man (Sybout Rewerda) dy't mei sjantaazje de geunsten fan in tsjep jongfaam (Christine) keapje wol. Christine en oaren binne ommers yn smokkelderij behelle. Yn it stik spilet in lotterijjoad, Pinto, in lytse rol. Hy praat Nederlânsk mei út en troch in ôfwykjende sinfolchoarder (heb ik een stoel

28. Werprinte as Nieske Stamsma. In forhael út 'e Greidhoeke. De Jouwer 1889. 
genomen en heeft Mozes ook een stoel genomen). Hjir hat de Joad gjin foarnamme, mar allinnich in (Spaansk-joadske) famyljenamme.

Yn It testamint fan Doeke Hendriks Zijlstra ${ }^{29}$ (1889) meitsje in merkereizger en in fioelspylder by in doarpsfeest mei in hurddraverij fan de gelegenheid gebrûk om yn te brekken by in boer. Yn in herberge biede se de bút (wat kostberheden en in bibel mei in testamint) oan de Ljouwerter Joad Maupie oan, dy't se earder troffen hiene. Mar dy wol it guod net ha. De fioelspiler bekent letter, yn syn eigen belang, de ynbraak. Hy hat by it stellen fan dat testamint útfûn dat hy sels as erfgenamt neamd wurdt. De Joad (Maupie) is wer minder ûnnoazel as de oaren: hy hat fuortdaliks de bedragerij yn it snotsje (oars as de herbergier). Hy praat 'Joadsk' Nederlânsk mei in opfallend soad jargon: Och myn ghoeje man, houje dood stil. ' $k$ Heb nog gheen dibbeltje te pakken de heele middag; och sjamaaie, as ik maar massels ('winst') maak. Hy wit ek mear fan de wrâld as in oar, as er de gek hat mei de fioelist: Kom Winiawsky, speul ons nou reis in moai stukje (Henryk Wieniawski, 1835-1880, wie in Poalsk-Joadske fioelist). De skriuwer spilet hjir mei de meartalige sitewaasje. Hy brekt mei opset sin mei de literêre konvinsje dat alle personaazjes inoar altyd fersteane, yn 'e folgjende dialooch tusken Maupie en de fioelspylder: M: Evengoed kan je met de kunst gesjochten weze. F: Hwette? M: Ik zeg dat 't beroerd is. En as Maupie de fioelspylder freget oft er miskien gjin Fries is, jout dy beskie: Hoe kinsto dat nou freegje, Joad? Maupie: Na, dat gheeft wat. Mijn vrouws broer spreekt Frans als de beste, en toch is zij op de Bhreedeplaats geboren - en nooit buiten ons landje gheweest. Op subtile wize wurdt hjir it tema fan de Joad as 'in frjemd' of 'de oar' beneamd.

De Joadske fiskferkeaper Levie út Hwa is yens neiste (1890) fan de Hallumer boer, feedokter en boargemaster Cornelis Douwes van der Weg is ien fan de meast libbene Joadske personaazjes yn de Fryske literatuer. De plot fan it stik is frijwat komplisearre en in bytsje surrealistysk. De famylje fan de skoarstienreager Lolke en syn frou Maeike krije op sinteklaasjûn samarynienen in fûnling presint. Yn in oare trie yn it ferhaal wol in deune boer, Rommert Habbenga, syn pleechbern fan in houlik mei in skoarstienreagersbern ôfhâlde. Levie wint stadichoan it betrouwen fan Lolke en Maeike en spilet yn beide triedden in 'stjoerende' rol op 'e achtergrûn. Hy wit it ien en oar oer it komôf fan it bern, want: Levie heit overal zijn broeders, omdat is

29. Piebenga neamt him Doeke Harmens Zijlstra. 
verstrooid over de geheele aarde de nasje. ${ }^{30}$ De titel fan it stik lit al trochskimerje dat de Joad hjir de wiere, barmhertige meiminske is. Syn waarm en wat nayf karakter wurdt ûnder oaren stal jûn yn syn wize fan praten. Hy praat in ryk soarte fan 'Joadsk Nederlânsk', dat - fansels - in fiktive keunsttaal is, mar wol in libbene yndruk makket. Hy brûkt formules as "weet ik het?", dy't minder stereotyp lykje as "bij mijn ziel" (ensafuorthinne) by oare skriuwers. Hy makket ek in soad gebrûk fan it kommunikative 'freegjende antwurd' (Van de Kamp \& Van der Wijk 2006, ynlieding) yn syn dialogen mei oare personaazjes ("Wat is dat?" - "Nah, wat of dat mag zijn?"). Dêrnjonken brûkt er persoanlike 'stopwurden', soks as voor z'n eigenzelvers, niks niemandal, d'r perlisje ('de politie') en de rijke nabob ${ }^{31}$. Syn gefoelslibben komt ta utering yn in oantal monologues intérieurs. Syn jongeseftige ûnskuld krijt foarm as er oer himsels yn 'e tredde persoan praat. (Wat op himsels net ûngewoan is by Joadske personaazjes, bygelyks by Kiemstra.) De skriuwer makket yn it stik gebrûk fan de twatalige sitewaasje. As fiskferkeaper bekflaait Levie letterlik mei syn klanten: waarom zalle ze (de fisken) niet nij zijn? Bij mijn lieve memmeke, anders zal ik nij zijn (hjir mei in retoarysk 'freegjend antwurd'). Letter yn it stik sil Levie op 'e nij Fryske wurden yn syn Joadsk idiolekt befrisselje, mar dan binne dy sitaten folle mear in blyk fan ferbûnens mei de skoarstienreagersfamylje: Weet ik 't? Has ('als') Levie profeteert ('profiteert') van het lok van jouë kindere, is dat brekken in lyts potsje; maar Levie weet, has dat 't hjoed is Sinderklaas. Yn omkearde rjochting nimt in Frysktalich personaazje (Doetsje) yn in monologue intérieur Levie's útspraak sjosjejaal oer.

Levie is yn dit toanielstik mear as allinnich in sympatike figuer. Hy wurdt hast in lid fan de famylje en sels ien fan de haadpersoanen. Hy hat in persoanlikheid en in karakter. Mar fan 'e oare kant bliuwt er wol 'de Joad' yn it stik.

\subsubsection{Njoggentjinde-iuwsk reälisme: proazawurk}

Hjerre Gjerrits van der Veen publisearre De kaertlizzer yn 1856 (werprinte yn 1965 en 1995). Yn dizze "stoarje der 19de ieu" lit in arbeider Obbe yn in petearke mei syn húsbaas it folgjende witte: Alles is negoasje, ju. (...) Simon

30. It wurd natie ferwiist yn dizze tiid net mear nei de heal-autonome Joadske mienskip yn Nederlân foar 1896, mar nei it Joadske folk yn it algemien (Van de Kamp \& Van der Wijk).

31. In nabob (klam op -bob) is oarspronklik in ûnderkening yn it ryk fan de Grut-Mogol; letter ek immen dy't yn Ynje ryk wurden is (WNT). 
Smouske hat my jister mar ris tige jild bean for myn touke, mar ik stean dy jeuden net rjucht. Hja slaen my to folle op hjar boarst as se swarre: "hik magh hier stokkeplind wurde" by dit en dat. Mar húsbaas jout him ta andert: Hja binne party al by 't rêd om of. Mar as se de slach om 'e earmtakke hawwe, de jeuden, den binne se tûzen parten better as de poepen.

De Hirddravery to Flearterp (1864) is nei alle betinken net troch Waling Dykstra skreaun (katalogus fan Tresoar), mar troch Tsjibbe Gearts van der Meulen (TDB, boarnelist WFT). Dy proazatekst giet yn 'e pleit tsjin gjirgens ("de woartel fan alle kwea" neffens de apostel Paulus), drankmisbrûk en sport (yn it bysûnder hynsteriden). De "jongehear Finael" is in trochbringer fan heech komôf. Syn mem besiket tefoaren te kommen dat oaren him jild liene, mar mei help fan in rike "soane Abrahams" sûnder namme of tekst wit er dat probleem út 'e wei te gean. It rint lykwols ferkeard mei de Jongehear ôf.

\subsubsection{It fiktyf Joadsk-Nederlânske etnolekt yn it 19de-iuwske reälisme}

De saneamde folksskriuwerij fan Waling Dykstra en syn tiidgenoaten soe mei it wurd reälisme better omskreaun wurde, sa't Abe de Vries (2015), mei rjocht, ha wol. In skaaimerk fan reälisme (en naturalisme) is dat de couleur locale en de eigen groepstaal omtinken krije. Je soene dan miskien ferwachtsje dat Joadske personaazjes op in natuerlike en reälistyke wize sitearre wurde. Dat is lykwols net sa.

Yn it foarste plak ha wy sjoen dat de measte Joaden yn Fryslân Stedsk en soms ek wol Frysk koene, dat wêrom soene dy hannelslju mei harren (potinsjele) klanten in aparte foarm fan Nederlânsk prate? Yn it twadde plak is it sabeare 'Joadske etnolekt' fan de Telenga-auteurs (Dykstra, Alma, Aartsma en Van der Weg) dúdlikernôch fan Hollânske foarbylden oernommen, sa't by Aartsma te sjen wie. Yn dizze paragraaf sil ek sjen litten wurde dat it sabeare Joadske praat betiden in humoristysk effekt ynhat. Mar dat nimt allegearre net wei dat de measte skaaimerken fan dat 'Joadske Nederlânsk' op 'e ien of oare wize wol mei de spraak fan besteande Joadske sprekkers te krijen hân hat. Dat is werom te finen yn Van de Kamp \& Van der Wijk en Hinskens, en foar in part ek by Winkler (dy't it 'Jodenhoeks' fan Amsterdam beskriuwt). Van Ginneken lit in soad tekstfragminten sjen, mar wurdt algemien as in net botte betroubere boarne besjoen. ${ }^{32}$

32. It ûntkomt Van Ginneken bygelyks dat it nijjiersferske fan 'Mozes Polak en Soon' in persiflaazje is (fl. Van de Kamp \& Van der Wijk, ynlieding). Alleman (1998:136f) 
De literêre Joadske keunsttaal by de fjouwer Telenga-auteurs en Kiemstra lit grutte oerienkomsten sjen. Dêrom rjochtsje ik my hjir benammen op dy fjouwer skriuwers. By de oare auteurs komme deselde skaaimerken foar, mar meastal mear beheind.

Alle skriuwers foegje $h$ 's ta oan it wurdbegjin: hik, hof, has ('als'). Aartsma, Alma en Kiemstra wikselje dy ôf mei ynfoege $h$ 's nei bylûden (hik dhoe), sa't de Halbertsma's en oaren ek dogge. Dy ynfoege $h$ 's meitsje in ûnwierskynlike yndruk, sa't hjirboppe (by de Halbertsma's) al sein is. Van der Weg wikselt de ynfoege $h$ 's krektoarsom ôf mei weilitten $h$ 's (hik $e b$ ), krekt as 'Mozes Polak en Soon'. Waling Dykstra is de iennichste dy't beide 'strategyen' brûkt, dat wol sizze sawol hik dhoe as hik eb. ${ }^{33}$

De staan-te-konstruksje, dy't by Van der Ploeg (1777) al foarkaam, komt by de Telenga-auteurs (en oaren) hieltyd werom, útsein by Waling Dykstra. Dy konstruksje liket net folle betsjutting te hawwen, mar wol in komysk effekt te berikken, benammen as it aspektuële tiidwurd staan mei in oar aspektueel tiidwurd (komen) kombinearre wurdt: je sthaen te wensche goeije dhag en dhenkje dat ik ben komme sthaen gek te worden.

De Jiddysk of Dútsk hearrende stimleaze ploffer yn it wurdbegjin ( $t u b$ beltje, hik pen) kaam by Van der Ploeg (1777) foar as in skaaimerk fan sabeare Jiddysk. By de lettere auteurs wurdt dat minder, sa't te ferwachtsjen wie. Mar it ferdwynt net hielendal. Alma brûkt noch that en keen ('geen') en Kiemstra brûkt than ('dan') en van thaag. Van de Kamp \& Van der Wijk en Hinskens binne spitigerôch net tige dúdlik op dit punt.

It hyperkorrekt lykjende zoebel en kibbesoeb (Hinskens, Van de Kamp \& Van der Wijk) komt by de Fryske auteurs mar ien kear foar, en wol by Alma: staande kijken, 'staan te kijken'. It is blykber wol in skaaimerk fan besteand Joadsk Nederlânsk, mar net fan fiktyf Joadsk Nederlânsk (Van de Kamp \& Van der Wijk, ynlieding: "Noem een woord met drie b's: kibbesoeb").

It sj-lûd fan 'sjaloom' komt foar yn sjiep ('skip') en sjosjeaal (Van der Weg), mar net yn wurden as sjtom en sjlim (dêr't it sj-lûd, fonotaktysk

skriuwt dat Van Ginneken "volgens Beem (1970) zelfs de toets van de milde kritiek niet kan doorstaan".

33. Men kin hjir net genôch beklamje dat it hjir om in persiflearjende keunsttaal te rêden is. Begripen lykas 'hyperkorreksje' of 'rule inversion' (alias 'regelomkering', sjoch Vennemann 1972) soene fertuten dwaan by in analyze fan in natuerlike taal (bygelyks histoarysk 'Joadsk Nederlânsk'), mar de relaasje tusken werklikheid en persiflaazje is no krekt by dizze sabeare 'aspiraasje' in riedsel (sjoch alwer Van de Kamp en Van der Wijk). 
besjoen, yn Joadsk etnolekt thús heart, lykas ek yn it Dútsk en Jiddysk, sjoch Van de Kamp \& Van der Wijk, ynlieding). Wy ha hjir, alwer, mei in fiktyf Joadsk etnolekt te krijen, dat ek by Hollânkse skriuwers foarkomt.

De ûntrûning fan foar-fokalen (frontal vowels) hat mei wissens in lang skoft karakteristyk west foar in Joadske útspraak fan it Dútsk en it Nederlânsk. It Jiddyske wurd Jiddisj (yn pleats fan Dútsk jüdisch) is op himsels al in moai foarbyld. Yn persiflaazjefoarm binne wy it al by 'Mozes Polak en Soon' (ca. 1770) tsjinkommen: hop de beers ('op de beurs'). Yn de Fryske literatuer komt it ferskynsel pas opsetten by de Telenga-auteurs, bygelyks Aartsma (hin de dheer, 'in de deur') Van der Weg (dibbeltje) en Dykstra (ghilde, 'gulden', ferheize 'verhuizen').

De ôfwikende wurdfolchoarder subject-verb-object (SVO) is likegoed in erfenis út it Jiddysk dy't sawol yn besteand as yn fiktyf Joadsk Nederlânsk oernommen west hat (en by Fritz Reuter, yn paragraaf 3.3): hik kom je staan te wensen goeie dag (Aartsma ${ }^{34}$ ). By Van der Weg is dat faak yn kombinaasje mei de bynwurden has dat: has dat jou bint een brave vrouw; maar has dat ik niet heb boodschap aan anderen. Dat op it each oerstallige has dat moat perfoarst in gefal fan fiktyf Joadsk etnolekt wêze. De auteur liket hjir in kontaminaasje te brûken fan (a) it Jiddyske ûnderskikkende bynwurd as ('dat') mei in komplemint-sin yn SVO-folchoarder, en (b) in gewoane Frysk-Nederlânske haadsin mei de dêrby hearrende SVO-folchoarder. De sin fungearret as in haadsin, wat it syntaktysk besjoen net is. It karakter fan kontaminaasje wurdt fersterke troch it oerstallige bynwurd dat, dat (op tautologyske wize) deselde betsjutting hat as Jiddysk as. Njonken de SVOfolchoarder komt ek VSO foar: $O$ waai me. heit me toen gemaakt die kerel een lawaai (Van der Weg). En by Bakker: Nah! Heeft hij mij gezeid "Nah, Pinto, neem een stoel"; heb ik een stoel genomen en heeft Mozes ook een stoel genomen. Dat dy VSO-folchoarder yn konsekutive omteksten te ferlykjen is mei "wandelt Sam door de Kalverstraat; ziet-ie Moos" liket my oannimlik ta, mar ik koe dat yn de literatuer net mei sa folle wurden weromfine.

De Telenga-auteurs ha njonken dy grammatikale ferskynsels ek in Joadske wurdskat yn de literatuer yntrodusearre, nei alle gedachten op grûn fan Hollânske foarbylden (bygelyks de saneamde Levie-Zadok-brieven fan Jan Schenkman, fl. Sanders 2017a, Meertens \& Wander 1958). Foarbylden binne moos ('jild'), goochem, kausjer ('koosjer, kauster') en sjovele

34. SVO is hjir net de meast gaadlike omskriuwing, mar de sin is de wjergader fan Hollânsk: Ik kom je goedendag wensen, mei OV yn 'e ynbêde VP. 
masematten ('minne keapwaar') by Aartsma; massels ('winst'), gannef ('smjunt'), gesjochte ('beroerd') by Zijlstra en een sjabbes ('sneinske') broek by Van der Weg. Utroppen lykas nah! ('hawar'), o waai ('earme ik') en bij mijn ziel hearre ta de gebrûklike wurdskat fan it fiktive Joadske etnolekt. By Aartsma, Alma en Van der Weg fine wy ek o sjorem magaaije! Wat dat betsjutte moat, is ûndúdlik, mar de útrop is offkomstich út in Hollânsk brulloftsfers (Joden: bruilofts-sermoen of sjorem! sjorem! Magaaije! $)^{35}$. Ut en troch is der ek in bewyske Frysk te finen yn it praat fan it Joadske personaazje, bygelyks een stikken jak en hin ' $e$ (' $y n$ ' $e$ ') foddesak by Aartsma. Fan Stedsk is net folle te merkbiten, ôfsjoen, miskien, fan inkeldris feul en seun, ${ }^{36}$ sjoch ek Van der Ploeg, yn paragraaf 3.2.

\subsection{De tweintichste iuw}

Krekt as by de njoggentjinde iuw it gefal wie (3.4) wurde toaniel (3.5.1) en proaza (3.5.2) hjir apart besprutsen. De taal fan de Joadske personaazjes krijt hjir gjin eigen kopke, mar wurdt en passant besprutsen.

\subsubsection{Tweintichste iuw, toaniel: Hollânsk, Stedsk en Frysk}

Foardat de Jongfryske beweging úteinset, begjint it byld fan de Joad yn 'e Fryske letteren (en benammen it toaniel) wat te ferskowen. Syn maatskiplike promoasje fan swalker en bûsesnijer nei jildliener hat yn 'e tiid fan it njoggentjinde-iuwske reälisme al plak fûn, sa't wy hjirboppe sjoen ha. In opfallende feroaring yn 'e tweintichste iuw is dat it personaazje ek net mear allegeduerich bryk praat.

It maatskippijkrityske toanielstik Maerteblom (1904) fan Yme Christiaans Schuitmaker hat oanlieding jûn ta in soad opskuor (Oppewal e.o. 274). In rike boer en fâd fan in jongfaam fergunt it famke har feint, in 'godloochener' fan ienfâldich komôf. It rint ferkeard ôf, hoewol't de Joad Aron de boer dêrfoar warskôge hie: doe sei de profeet: "gij zijt die man" (2 Samuel 2; mei oare wurden: jo binne sels dy wrede hearsker). Yn 'e húskeamer fan de boer wurdt by it miel foarlêzen Matteüs 6:24: "niemand kan twee heren dienen, God en den mammon". It is de earste kear - by myn witten - dat in Joadsk personaazje Frysk praat yn in toanielstik. It is oars

35. Sjoch Meertens, Nederlandse Liederenbank, koade Lbl KB Wouters 13051, "negentiende eeuw".

36. Mar sokke $e u$-lûden (yn pleats fan Standert-Nederlânsk oo) komme yn streektalen yn Hollân ek wol foar: stedsk feugel, holl. veugel (Van Bree \& Versloot 2008: 115). It wurd stikken kin yn alle gefallen net stedsk wêze (ibidem, 118). 
wol in Frysk mei bewyskes Hollânsk tuskentroch (in keardel met een hart), dat miskien suggerearje moat dat Aron it Frysk as twadde taal leard hie.

It is ek foar it earst dat it Joadske leauwe sels, yn 'e foarm fan in sitaat út it Alde Testamint, mei klam yn it stik behelle wurdt; dat sitaat (fan Aron) wurdt hjir oanfolle mei ien út it Nije Testamint (by de boer thús). De Joad yn it stik lit syn eigen wiisheid skine, krekt as by Van der Weg, en is sadwaande wer 'oars' as de oaren.

Libbensstoarmen (1913) fan R. Zeilstra hat net folle om 'e hakken. It wie "troch in ienfâldich man skreaun" (foaropwurd fan S. van der Zee). It spilet him, net tafallich, of yn de taapkeamer fan "Van Pimpelen", want it giet oer drankmisbrûk. In "Abreham Cohen, brillejoad" spilet in lytse rol (Nu zal ik $u$ komen te vrage, kan ik u verkoope een bril?). Hoewol't ien fan de oanwêzigen te witten docht dat er syn nocht hat fan "dy smoarge smous", is dy lêstneamde perfoarst net de smjunt yn it stik. Maar nu zal ik jo kome vrage. Kom jij ook wel eens in de stad? Hebje dat grote boevehuis wel eens gezien? Hoeveel jode zitte daar in? Hoeveel jode zitte in Veenhuize? ... Hoeveel jode-kinderen worden buiten de echt gebore? Hjir hat men sûnder mis de emansipaasje en it selsbewustwêzen fan de Joaden by de ein. By dit byld past de Joadske húsdokter Aletta Jacobs fan Sappemeer (frouljuskiesrjocht 1917) better as, bygelyks, de ienfâldige feekeapman Salomon Levy fan de Westereen (Kollumer Oproer 1797), waans biografy foar it grutste part út stikken út it plysje-argyf gearstald is (fl. Vries 2019). Abreham is hjir ek dejinge dy't op 'e hichte is fan de polityk yn Den Haach (in wetsfoarstel oer sterke drank). Syn spraak is wer it (fiktyf) Joadsk Nederlânske taaltsje, mar de klisjeemjittige $h$ 's binne weibleaun. Wol seit er hieltyd, by it opkommen: Goeien tach, mensen.

Johannes A. Noordenbos syn stik Wiste it noch? (1918) is twa kear werprinte (1927, 1930). It giet oer in boer mei skulden, in tema dat hjirboppe en rûnom yn de Fryske literatuer faak en folle werom komt. De jildliener is dan geregeld in Joadske lommertsman, en dat is hjir ek it gefal. Simons (hy hat wer allinnich in famyljenamme, gjin foarnamme) lient de man tsjin tolve persint. Hy is gjin freonlike figuer ("in echte joad, stobbelburd, klean rom grut") en de skelwurden ("smoarge joad", "ferflokte joad") binne op it toaniel goed te hearren. Brouwer (1931) neamt dit stik yn syn lêzing as foarbyld fan in "negatyf byld" fan de Joad. Fan 'e oare kant wurdt yn it toanielstik sein dat er neat misdiedichs docht. Hy praat yn it stik Stedsk: Dat niet, mar ik must mien geld maar weerom hebbe. Je geve het as je it hè. De kontemporêne besprekkers (Douwe Kalma, R.W. Canne) ha gjin miening oer de rol fan de Joad, mar wol oer it minne Frysk fan it stik. 
Sybe Douwes de Jong syn stik Frâns Winia (1930) giet oer in ûnderstelde trijehoeksferhâlding tusken in jonge boerinne, in jongfeint en in dokter. It toanielstik wurdt priizge om syn knappe psychologyske tekening fan de haadpersoanen. Wannear't de jonge boerinne (Tine), twongen troch de jildpine, har kostberheden ferkwânselt, komt de Joadske jildliener Nathan Levison op 'e planken. Hy praat Stedsk en hy is in freonlike figuer dy't goed mei Tine har âlden koe: Sa'k der mar inkomme, mefrou? Noch gesond? (...) It is oek al een hele tiid leden dat ik mefrou sien hef. Wy doene niet veul saken met elkaar teugenswoordich (sûnt de dea fan Tine har âlders). Ik waer der dan oek oates en toates. As groat jonge al. As ik op de plaets kaem om peardehaer, bonken of wol, ik dee in dy tiid in alles, mut mefrou rekene, den waer it altiten: Nathan ek in bakje kofje? Soa echt hartlik. Dat fine je niet feul. Tine tinkt fan har kant mei waarmte werom oan 'e joadekoeken dy't se eartiids by dy besiten krige. Nathan Levison is in like libbene en waarme persoan as Levie yn Hwa is yens neiste fan Van der Weg.

\subsubsection{Tweintichste iuw, proaza (Kloosterman en Brolsma)}

Yn 'e tweintichste iuw nimt de roman, it sjenre fan de ynternasjonale literatuer, de rol oer fan de rimen, teltsjes, fersen en sketsen út de njoggentjinde iuw. De Jongfryske beweging sil nei de Earste Wrâldoarloch op it toaniel ferskine. Wichtige boarnen foar dit ûndersyk binne Simke Kloosterman en Reinder Brolsma, twa skriuwers dy't, tafallich of net, nei de Twadde Wrâldoarloch beskrobbe waarden om har maatskiplike hâlding (Zondergeld 1978, Riemersma 2006, Sijens 2001). ${ }^{37}$

Yn it wurk fan Simke Kloosterman komme in stik as wat Joadske personaazjes foar, dy't noait folle mear te betsjutten ha as in stereotype singeliere bûtensteander. It ferhaal 'Beppe yn 'e fleur' is yn 1897 skreaun, opnommen yn de bondel Ruth, in hânfol ieren (1910) en werprinte yn De gielguorde II (1944). In "kiezzejoad, sa's yn dy tiden folle op "e marken to finen wierne" bringt op "e merk yn Dokkum mei geraas en maneuvels syn "nieuwste vinding der wetenschap" oan de man. (De lêste literêre kiezzejoad foar Kloosterman wie better net te witten dy fan Pibomme by de Halbertsma's.) "Hy haspele, lyk as allegear fen 'e joadske neiteam, nuver om mei de $h$ en stammere sims bryk oer in frjemd wird hinne, mar de ienfâldige lju mienden, dat soks brûktme by de learden wier, en hy hie in bulte harkers en

37. Kloosterman, yn Spreuken 1962, nr. 475: Doch noait saken mei Joaden Noait. Sijens 2001 jout in balânsearre besprek fan Brolsma syn wurk en libben. 
keapers." In grutte keardel tsjinnet him as frijwilliger oan. "Een lelijk geval", sei Abraham's soan, "daar zulle we je fluks af helpe, vrind. Sam, m'n jong (syn soan), kom mal hier." (Mal is hjir gjin printflater, mar it Dútske en Jiddyske '(ein)mal'). Dit beteart mis en it hynder fan de Joad rekket op 'e kletter, wylst de man neat oars rôp as "Sara, Sara, sta je Levie bij!”. Mar Sara siet yn hjar jern- en bânwinkeltsje to Ljouwert en hearde sadwaende syn kriten net. En Sam, dy ondogenske jonge, dêr't al in keapmansholle op siet, gûlde mei syn skel joadejonges-lûd boppe alle minsken út: "Help, help, slúge Dokkumers. Wat 'en kostelike aanbeveeling voor me vader! Tien centen, die 't peerd houdt.". Ofsjoen fan de klisjees ("Abraham's soan") en it "omhaspeljen mei de $h$ " falt jin de taalwiksel tusken de heit (fiktyf Joadsk Nederlânsk) en de soan (Stedsk) op.

Kloosterman har koart ferhaal 'Syn libbenswinsk' is, krekt as 'Beppe yn 'e fleur', yn 1897 skreaun en yn Ut 'e gielguorde II (1944) publisearre. It giet, op 'e nij, oer it kwea fan de lotterij, dêr't in jongeman út it heidegebiet (Jochem) him net tsjin ferwarre kin ("de jilddivel naem Jochem yn 'e maling"). Hy sil nei de stêd ta om in lot te keapjen. Sa tinzende wei kaem er by in foech keammerke en dêr siet Sammelsen ('Samuelson') al op in hege skammel foar it ljocht, en al ringen wier in briefke ynwiksele tsjin syn sûrfortsjinne gounen en Jochem trêdde mei in gerêst moed nei hûs ta. Jochem sil in fikse priis winne, mar syn hert ferstiennet, en hy komt te ferstjerren. Doarp, ienfâld en soberens (syn frou Wemeltsje, wers fan de lotterij) tsjin stêd, ferlieding en ûngelok (Jochem) oer.

Yn 'e roman It jubeljier (1927) fan Simke Kloosterman is it yn 'e jachtweide libben en drok. [K]oumelkers, ielfiskers; en dêrtwisken yn prizen de joaden mei in heech noaslûd harren negoasje oan: kasjmiren en bûntsidene doeken, knipkes mei kralene taskes; hinneptou, spikers, hazzesprongen, nije helters, pipekoppen, folle net genôch, hwat se bêst slite koene oan de lju, $d y$ 't sims by 't winter gjin wiken of moannen fan it hiem kamen. Mar letter yn it ferhaal moetet de lêzer in Joad fan in oare maatskiplike klasse. It is Jozua de Metz, feekeapman út Amsterdam. Hy "bitelle graech goed jild foar goed gûd”. Hy praat Frysk (Wer hielendal de âlde, Haeije?), mar de lêzer sil begripe dat de Amsterdammer Jozua gjin Afûk-kursus folge hat: de literêre konvinsje is hjir feroare. De sprekker wurdt net mear altyd (lykas yn de Ljouwerter Krante) yn syn eigen taal sitearre, likemin as letter (nei alle gedachten) it gefal is yn it koarte ferhaal 'Frou Mesker' fan Geart Jonkman út 1960. De couleur locale wurdt yn Kloosterman har roman oars wól foar kar nommen as, earder yn it ferhaal, in pear Stedskpraters oan it wurd binne (dou muste soa mar denke). 
Daniël fan Kuken, de haadpersoan yn Reinder Brolsma syn roman Groun en minsken (1940), wint in hynder by in lotterij. Syn heit is derop tsjin, mar keart de saak net op. De Joad is "kreas klaeid" en wurdt omskreaun as "it Fortún yn "e persoan fan de lotten suteljende stedman". Yn dit ferhaal is de lotsjeferkeaper, lykas yn mannich oar wurk, net mear as in ynstrumint yn in fertelling oer in oar syn habsucht en ûnnoazelens. Letter rydt Daniël syn soan Lammert op syn hynder by fersin hast tsjin it "spektakelstik yn oanbou" fan de Joad Bontallé oan. Hui wat wurdt dy Joad kjel, wat in noed foar syn negoasje as hy eangstich ropt: "Hou him, hou him dochs goëed, jonkje". Lammert ropt him ta: "[s]moarge Joad"[.] Hui nee, dat is net goed fan Lammert, want de Joad is ûngelikens folle earliker yn hannel en wannel as de glûperteftige hynsterosser (dy't it hynder oan Daniël ferhannele hie). Bontallé praat hjir Stedsk, in taal dy't Brolsma, auteur fan de kranterubryk 'Gesprekken op de brug', goed yn 'e macht hie. (Op side 28 praat in personaazje "ûnforsteanber plat Grinzersk": hy seit bygelyks Frijsland. ${ }^{38}$ )

Gearfetsjend kinne wy sizze dat nei 1900 de oergong fan it fiktive Joadske ideolekt nei besteand Hollânsk, Frysk en Stedsk syn beslach krijt, sawol op it toaniel as yn proaza. By Kloosterman (krekt foar 1900) liket it "omhaspeljen" mei de $h$ fan de merkeman de stereotype beskriuwing fan it Joadske personaazje te ûnderstreekjen, mar syn soan praat 'al' Stedsk. Omdat dyselde taalwiksel ek yn Waling-Dykstra-eftige toanielstikken nei 1900 te sjen is, lykje de literêre streamingen gjin beskiedende faktor te wêzen. It leit mear yn 'e reden dat de maatskiplike werklikheid (de Joadske emansipaasje) dy ferskowing yn gong set hat.

De auteur Brolsma liket fierders folle minder anty-joadsk te wêzen as de auteur Simke Kloosterman. Nei de Twadde Wrâldoarloch krige de persoan Brolsma in publikaasjeferbod (Kloosterman wie al yn 1938 ferstoarn). Krekt as de persoan S.D. de Jong, hoewol't de auteur S.D. de Jong (fan it toanielstik Frâns Winia) in sympatyk Joadsk personaazje op 'e planken set hat. Auteurs en persoanen binne hjir net deselden.

\section{Besprek en konklúzjes}

Yn it boppesteande oersjoch docht bliken dat sawol it Joadske personaazje as syn wize fan praten yn 'e rin fan 'e tiid feroarje.

Yn 'e tiid fan Van der Ploeg en letter by de Halbertsma's is de Joad in omswalkjende earmelijer. Hy makket him no en dan skuldich oan geweld en dieverij. Yn 'e njoggentjinde iuw komt er minder fier fan de doarpsmienskip

38. Ferlykje Ter Laan, Nieuw Groninger woordenboek 1929, Vrais, Vraislaand. 
ôf te stean. It giet der net mear om dat er ûnbetrouber wêze soe, mar folle mear dat er sa besjoen wurdt troch dizze en jinge. Wol bliuwt er hieltyd in type. Dat komt benammen ta utering yn syn praat. Yn 'e rin fan 'e njoggentjinde iuw kliuwt er op 'e maatskiplike ljedder omheech: de marseman fan eartiids wurdt in jildliener. By Brolsma, yn 'e tweintichste kin er sels in frijwat eptige pommerant wurde.

Tagelyk mei it karakter feroaret ek syn taal. By Van der Ploeg praat er sabeare Jiddysk (mongen mei Hollânsk, Frysk en miskien wat Stedsk). By de Halbertsma's wurdt dat in tige klisjeemjittich Hollânsk mei ynfoege $h$ 's (bhen je mhal). De let-njoggentjinde-iuwske toanielskriuwers út it fermidden fan Waling Dykstra nimme in fiktyf Joadsk etnolekt út Hollânske boarnen oer. Dat taaltsje hat in syntaksis, in wurdskat en in retoryk dy't earder net yn 'e literatuer foarkaam. Yn 'e tweintichste iuw praat it Joadske personaazje in besteande taal (Hollânsk, Stedsk, Frysk) mei út en troch in bewyske fan Joadsk aksint.

Literêre streamingen en sjenres lykje hjirby gjin faktoaren fan belang te wêzen, mar de (mei fertraging reflektearre) maatskiplike werklikheid nammerste mear. Joadske persoanen emanipearje har yn 'e rin fan 'e tiid en nimme de taal fan haren omhjouwing oer. Literêre faktoaren spylje oars wol in lytse byrol yn it gehiel. Yn in moderne roman kin in haadpersoan bygelyks de taal fan it boek (i.c. Frysk) prate, hoewol't er sels oarstalich is (sjoch Brolsma). De politike of maatskiplike hâlding fan de skriuwer liket ek minder fan belang te wêzen as men miskien ferwachtsje soe (sjoch 3.5.2).

Dat it taaltsje fan de Joadske sprekkers in fiktyf taaltsje is, mei dúdlik wêze. Foar de measte boarnen (Brugmans \& Frank 1940, Alleman 1989, Groeneboer 1995, Sanders 2017a, b) is dat evidint, dat se dogge net folle war om it te bewizen. Mar je kinne der wol in smeet op dwaan. It sabeare Jiddysk by Van der Ploeg mist de syntaktyske en fonologyske ferskynsels (wurdfolchoarder, ûntrûning) dy't karakteristyk binne foar besteand Jiddysk en Joadsk-Nederlânsk, bygelyks. Boppedat wurdt it taaltsje (krekt as by lettere auteurs) dúdlikernôch foar in komysk effekt brûkt.

Halbertsma's bhen je mhal is oars net as in sjabloon. Hjirboppe is al útlein dat benammen dy tuskenskode $h$ 's in ûnwierskynlike yndruk meitsje en allinnich yn Nederlân as karikatuer lykje foar te kommen. Oer it Fiktyf Joadsk Etnolekt fan de reälisten is folle mear te sizzen. Yn it foarste plak meie wy oannimme dat Joadske sprekkers yn dy tiid folle better de taal fan har omjouwing sprutsen (paragraaf 2.1). Yn it twadde plak is dat sabeare Joadsk-Nederlânsk fan Hollânske foarbylden oernommen, sa't hjirboppe oantoand is (paragraaf 3.4.1). In soad taalkundige en leksikale eigenskippen 
fan dat etnolekt soene je by eardere auteurs ek al ferwachtsje. Boppedat is dit etnolekt net botte homogeen. Der binne bygelyks trije 'strategyen' om mei ynskode en/of weilitten $h$ 's om te tyspeljen. It personaazje by Zijlstra brûkt wurden dy't oare personaazjes net kenne (bygelyks gannef en gesjochten). De auteurs lykje de grammatika fan in besteand Joadsk etnolekt ek net hielendal te begripen (bygelyks has dat mei haadsin, it foneem $s j$ op ferkearde plakken, ensafuorthinne). De útdrukking staan te wurdt, foar in komysk effekt, folslein oerstallich brûkt en mist syn aspektuële betsjutting. Net alle skaaimerken fan Joadsk Nederlânsk komme yn it fiktive ideolekt foar en oarsom (bhen en mhal lykje folslein keunstmjittich). Van der Kamp en Van de Wijk jouwe it foarbyld fan de 'Joadske' útspraak fan de $r$, dy't (sa't se skriuwe) op it Fryske plattelân grif in nuvere yndruk makke hat. Dêr sjogge wy yn de literêre teksten lykwols neat fan werom.

Oer it karakter fan de Joadske personaazjes sizze Van der Woude \& De Haan (1995:19) dat de Joad "in het volkstoneel" as in "karikatuur" ôfbylde wurdt, en dat er "een niet helemaal eerlijke venter van textiel of loterijbriefjes" is dy't "vee of waardevol antiek voor een (te) zacht prijsje wil opkopen" en "zich bedient van een gebrekkig uitgesproken Nederlands of Stadsfries" (sjoch it sitaat yn "e ynlieding, paragraaf 1). Yn rûge halen is dat wier, sa't wy hjirboppe sjoen ha, mar dat "stereotype beeld" fan dy sjaggeljende keapman is no krekt folle minder algemien as Van der Woude en De Haan ha wolle, oars as wat it taheakke sitaat fan Aartsma tinke lit. Boppedat docht dudlikernôch bliken dat de Telenga-auteurs, njonken it fiktive Joadsk Nederlânsk etnolekt, ek in mear posityf klisjee-byld oer Joaden út Hollân oernommen ha (ferlykje Weijtens 1971:38-43).

Jelle Brouwer (1931) hat in wat positiver oardiel as Van der Woude \& De Haan. Hy brûkt gjin wurden lykas "stereotyp" of "karikaturaal", mar hy freget him wol off oft de personaazjes "nei it libben tekene" binne. Fierders falt him op dat mannich personaazje yn "e njoggentjinde en tweintichste iuw in sympatike yndruk makket (bygelyks by Dykstra, Zijlstra en De Jong), yn tsjinstelling ta it Fryske taalgebrûk (joadeguod, joadestreek) en oars as dat yn de klucht Mayke Jakkelis fan 1777 it gefal wie. Boppedat sjocht Brouwer oare details as Van der Woude en De Haan: de Joaden binne op it toaniel hieltyd reizgjende keaplju ("yn sahwet alles") dy't de "nijtsjes út 'e grutte wrâld" opheine, en dêrmei ymplisearret Brouwer fansels: oars as de doarpske boer yn it toanielstik. Ek hiene hja faken in soarte fen philosophyske visy op it libben, obstrewearret er. En: Hwerom b.g. komt de joad sa faek foar yn Fryske toanielstikken? As de skriuwer in priis út 'e lotterij as ûnderwerp fen syn toanielstik naem, den hie er in joad nedich. Moast der op 
de planken hwet forhandele wirde, den wier de joad hjirfoar in tige tankber type. As er de ljue om krom en bryk praten ris laitsje litte woe, den moast der in joad op it toaniel komme.

Dat de Joad hieltyd in "karikatuur" is en yn 'e regel yn in "stereotyp beeld" beskreaun wurdt, is lykwols net te ûntstriden. Dat feit hat, mear as terjochte, grutte argewaasje jûn by Joadske en net-Joadske ûndersikers yn dizze tiid (ûnder oaren Boas, Groeneboer en Sanders 2017a). Wêr't hjir de oast sit, is net de fraach oft de útbylde Joad sympatyk oerkomt of net, mar oft er as yndividu útbylde wurdt of as type (sjoch ek Weijtens 1971:38-43). Yn in pear gefallen, benammen by Van der Weg en De Jong, wurdt de Joad àl in waarme persoanlikheid fan fleis en bloed. Mar dat is mear útsûndering as regel.

It ferskil yn persepsje tusken Brouwer en Van der Woude \& De Haan hat sûnder mis mei de tiid te krijen: foar en nei de Twadde Wrâldoarloch. Mar wierskynlik ek mei it perspektyf fan de eigen groep en ûnbekendheid mei de oare. De poerbêst dokumintearre bondel fan Vriesema e.o. (2006) oer "nieuwkomers in de provincie door de eeuwen heen" besprekt de ymmigraasje fan Joaden, lapkepoepen en hantsjemieren yn in provinsje dêr't gjin wurd Frysk praat waard, sa't it liket. ${ }^{39}$ Yn 'e Fryske literatuer kommunisearje frjemd en eigen, krektoarsom, sûnder problemen yn 'e eigen taal meiinoar. Mar hoe wie it yn werklikheid? Neier ûndersyk nei sokke kwestjes soe net oerstallich wêze.

Dit artikel giet, by einbeslút, oer literatuer. Sa't yn de ynlieding sein is, kinne skriuwers op in kreätive wize gebrûk meitsje fan it ferskaat oan talen yn har wurk. Van der Weg hat syn Levie net allinnich in folslein persoanlik idiolekt jûn, mar ek in meifielend karakter, dat yn 'e foarm fan Fryske 'sitaat-wurden' stal krijt. It hást perfekte Frysk fan Aron yn Schuitmaker syn Maerteblom is dêrmei te ferlykjen. Zijlstra brekt mei sin mei de konvinsje dat alle personaazjes inoar oer taalgrinzen hinne fersteane. De taharker sil him bewust wêze fan it Droste-effekt ${ }^{40}$ as in goekunde fan de boer him as Levi Smoel foardocht yn Alma syn stik. Dy goekunde wurdt eefkes letter op 'e planken priizge om syn goed slagge imitaasje fan in sabeare etnolekt, dat bûten it toaniel hielendal net bestiet. Zijlstra (It testamint, 1889) en de auteur fan Mayke Jakkelis, 1777 (yn in boppeneamde

39. Dit is net it iennichste blyk fan ûnbegryp yn Vriesema e.o. Mieren binne gjin eamelders, bygelyks. Foar Beem jildt dit beswier perfoarst net.

40. In Droste-effekt yn 'e literatuer is bygelyks in boek oer in biograaf dy't oer in skriuwer skriuwt. It tanket syn namme oan de bekende blikjes Droste's cacao, mei in ôfbylding fan in sikesuster dy't in blêd mei krektsa'n blikje tôget. (Sjoch etymologiebank.nl) 
passaazje oer 'species', paragraaf 3.2) brekke mei opset sin mei de konvinsje dat personaazjes inoar oer taalgrinzen hinne fersteane.

Sokke literêre boarterijen meie op de Fryske lêzer in fertroude yndruk meitsje, mar se soene likegoed in plakje yn in mear wiidweidige stúdzje fertsjinje. Der bestiet bûten Nederlân wol wittenskiplike stúdzje oer sokke ferskynsels, ferlykje Slofstra $2015 .^{41}$ Sok ûndersyk soe net allinnich goed passe yn it ramt fan de frisistyk as minority studies, mar ek de Fryske literatuer in rol jaan dy't er fertsjinnet. Dat wol sizze: de rol fan in literêr 'universum' dat net allinnich yn in meartalige maatskiplike kontekst bestiet, mar ek sels de kontekst is fan in meartaligens, dy't ommers net sljochtwei in maatskiplik feit is, mar ek in literêr konstrukt.

Bottemaheerd 7

9737 NA Groningen

boukeslofstra@hotmail.com

\section{LITERATUER}

Aartsma, Klaes 1862. It tsiende gebod, of: sa komme de slînders to pas! Kluchtspil mei sang in twa bedrieuen, opmakke troch Kl. Aartsma Az. Frjentsjer, Telenga.

Alleman, Colien 1989. De Jood in de literatuur van de zeventiende en achttiende eeuw: een onderzoek naar stereotypen in toneelstukken en proza. Amsterdam (eigen útjefte).

Alma, Hermanus 1863. Trye jieren letter of Levi Smoel, de Lotteryjoad. Toneelstikje în twa ûtkomsten, troch in lid fen it Sangselskip "Nut en Genoegen" to Menaam. Frjentsjer, Telenga, 1863.

Bakker, H.E. 1885. In rôt yn 'e stap. Blyspil mei sang yn 5 bidriuwen. Apeldoarn, Hingst.

Beem, H[artog] 1953. 'Ut de skiednis fan de Joaden yn Fryslân.' De Tsjerne 8, 366-377.

https://dbnl.org > tekst > tsj002195301_01 > tsj002195301_01_0105

Beem, Hartog 1967. Resten van een taā. Woordenboekje vān het Nederlandse Jiddisch. Assen, Van Gorcum.

41. Oer ferlykbere saken yn ûnder oaren De sûnde fan Haitze Holwerda en it 'hispanic' toaniel yn 'e Feriene Steaten. 
Beem, Hartog 1974. De Joden van Leeuwarden. Geschiedenis van een Joods cultuurcentrum. Assen, Van Gorcum (Fryske Akademy, nr. 468).

Besten, Hans den 2006. 'Jiddisch Hebreeuws in Nederlands en Bargoens'. (nei oanlieding fan J.G.M. Moormann, De geheimtalen (...) 2002. Tijdschrift voor Nederlandse Taal- en Letterkunde 122, 233-259. https://www.dbnl.org/tekst/_tij003200601_01/_tij003200601_01_0018.php

Besten, Hans den (†) 2010. Eikel, nozem, piegem en andere Jiddisch-Bargoense etymologieën. Tijdschrift voor Nederlandse Taal- en Letterkunde 126, 394-415. https://www.tntl.nl/index.php/tntl/article/ download/ 7/224

Birnbaum, S.A. 2016. Yiddish: A Survey and a Grammar, Second Edition. With new introductory essays by David Birnbaum and Eleazar Birnbaum, Kalman Weiser, and Jean Baumgarten. Toronto University Press.

Blom, Hans \& David Wertheim \& Hetty Berg \& Bart Wallet (red.) 2017. Geschiedenis van de joden in Nederland. Amsterdam, Balans.

Boas, Henriette 2000. 'Belletrie als bron', in: J. Bethlehem, F. Hiegentlich, F.J. Hoogewoud (red.), Gids voor onderzoek naar de geschiedenis van de joden in Nederland (Amsterdam: Schiphouwer en Brinkman, 2000), 7396.

Brandsma, W.L. (ed.) 1951. útjefte fan Feike Hiddes van der Ploeg, Mayke Jakkelis. Magnus-rige, teksten út de Fryske skriftekennisse nr. 5, Fryske Akademy, nr. 56. Boalsert, Osinga.

Bree, Cor van \& Arjen P. Versloot 2008. Oorsprongen van het Stadsfries. Met medewerking van Rolf H. Bremmer. Ljouwert, Fryske Akademy.

Broekema, Pauline 2001. Benjamin. Een verzwegen dood. Amsterdam, Arbeiderspers.

Brolsma, Reinder 1940. Groun en minsken. Snits, Brandenburgh.

Brouwer, Jelle H. 1931. 'De Joad yn 'e Fryske toanielstikken'. Anonym besprek fan Brouwer syn lêzing foar de krite Ljouwert fan it Selskip fan Fryske Tael- en Skriftekennisse, Leeuwarder Courant 21-01-1931 (www. dekrantvantoen.nl).

Brugmans, H. \& A. Frank (red.) 1940. Geschiedenis der joden in Nederland. Amsterdam, Van Holkema \& Warendorf.

Buma, Wybren Jan \& Gerbenzon, Pieter \& Tragter-Schubert (red.) 1993. Codex Aysma. Assen, Van Gorcum.

Burger, H. 1944. Avondrood. Bloemlezing en overzicht der Stadfriese, Amelandse en Bildtse letteren. Assen, Van Gorcum.

Codex Aysma: sjoch Buma e.o. 1993.

Dykstra, Klaes 1977. Lyts hânboek fan de Fryske literatuer. Ljouwert, De Tille. 
Dykstra, Waling 1851. De toalfte Maye. In: Winterjounen by Gealeboer. Frjentsjer, E. Ippens.

Dykstra, Waling 1891. Op Maeijedei by Wigle Kwânseler: kluchtspil mei sang. Holwert.

Dykstra, Waling 1904. 'In kristen en in joad'. Fers yn De Fryske húsfrjeon, rym en ûnrym út earder en letter tiid fen Waling Dykstra, Ljouwert, R. van der Velde, 129-133.

Dykstra, Waling 1912. 'In trien'. Fers yn 'e bondel Net ny en ek net wei, rym en ûnrym út earder en letter tiid fen Waling Dykstra, diel I. Ljouwert, Eisma, 69-70. Ek ôfprinte yn besprek fan Cornelis Wielsma, Leeuwarder Courant 16-09-1912 (www.dekrantvantoen.nl).

Endt, Enno \& Lieneke Frerichs 1972. Bargoens Woordenboek. Kleine woordenschat van de volkstaal. Amsterdam, Erven Thomas Rap.

Feitsma, Anthonia 1956. Frysk út de 17de ieu, teksten en fragminten. Utjown fan A. Feitsma, mei in foaropwurd fan prof. dr J.H. Brouwer. Estrikken nr 15, Grins, Frysk Ynstitút.

Ginneken, Jac. van 1914. Handboek der Nederlandsche taal, deel II: De sociologische structuur (pp 2-102: De Jodentaal). Nijmegen.

Goudsmit, Sam 1918. Zoekenden. Amsterdam, Nederlandsche bibliotheek (twadde printing). https://www.dbnl.org/tekst/goud005zoek01_01/ goud005zoek01_01_0001.php

Groeneboer, Joost 1995. 'Joodse personages op het Nederlandse toneel. Joodse stereotypen en hun aantrekkingskracht op het publiek'. In: J. Groeneboer \& H Berg (eds.), Dat is de kleine man: 100 jaar Joden in het Amsterdamse amusement, 1840-1940 (pp 117-143). Amsterdam-Zwolle.

Gubser, Martin 1998. Literarischer Antisemitismus. Untersuchungen zu Gustav Freytag und anderen bürgerlichen Schriftstellern des 19. Jahrhunderts. Göttingen, Wallstein.

Halbertsma, J.H. 1829. De reis nei de jichtmasters, It Bolserter Nut en De âld kies fen Pîbomme (letter bondele yn Rimen en teltsjes).

Halbertsma, J.H. 1839. Grouster merke (bondele yn Rimen en teltsjes).

Halbertsma, J.H. 1840-5. Letterkundige naoogst (twee delen). Dimter, J. de Lange (sjoch ek: De Jong \& Slofstra).

Hinskens, Frans 2015. Wijdvertakte wortels. Over etnolectisch Nederlands. Amsterdam University Press. https://oapen.org/download?type= document \&docid $=636090$

Hofmann, Dietrich \& Popkema, Anne Tjerk 2008, Altfriesisches Handwörterbuch. Heidelberg, Universitätsverlag Winter. 
Horch, Hans Otto 1985. 'Judenbilder in der realistischen Erzählliteratur. Jüdische Figuren bei Gustav Freytag, Fritz Reuter, Berthold Auerbach und Wilhelm Raabe'. Yn: Herbert A. Strauss \& Christhard Hoffmann (Hrsg.), 140-171.

Horst, J.M. van der 2008. Geschiedenis van de Nederlandse syntaxis. Leuven University Press.

Houten, Ulbe van 1938. De sûnde fan Haitze Holwerda. Dokkum, Kamminga.

Jacobs, Neil G. 2005. Yiddish: A Linguistic Introduction. Cambridge University Press.

Jensma, Goffe 1998. Het rode tasje van Salverda. Burgerlijk bewustzijn en Friese identiteit in de negentiende eeuw. Ljouwert, Fryske Akademy.

Jong, Alpita de \& Bouke Slofstra (eds.) 2017. J.H. Halbertsma, "A plank I saved from a shipwreck." Halbertsma's Commentary on T. Hemsterhuis's Lectio publica de originibus linguae Graecae. Amsterdam, Stichting Neerlandistiek VU; Münster: Nodus.

Jong, Alpita de 2018. Triomfen en tragedies van een uitmiddelpuntig man. Joost Halbertsma 1789-1869. Een biografie. Grou, útjouwerij Louise.

Jong, Sibe Douwes de 1930. Frâns Winia. Snits, Brandenburgh.

Jonkman, Geart 1960. 'Frou Mesker'. Ferhaal yn De Tsjerne 15, 37-41. https://dbnl.org/tekst/_tsj002196001_01/_tsj002196001_01_0013.php

Jonkman, Reitze J. \& Arjen P. Versloot 2008. Tusken talen. It ferhaal fan de Fryske taal. Ljouwert (Leeuwarden), Afûk.

Van de Kamp, Justus \& Van der Wijk, Jacob 2006. Koosjer Nederlands. Joodse woorden in de Nederlandse taal. Amsterdam, Contact.

Kiemstra, R. 1862. 't Kjeallefel en 't lotterybriefke. Kluchtspil yn twa bedrieuwen. De Gerdyk, A.G. Zwart (Selskip Halbertsma Warga). https://books.google.nl/books/about/T_Kjeallefel_T_lotterijbriefke_Kluchtspi. html?id=pCpdAAAAcAAJ\&redir_esc=y

Kloosterman, Simke 1910. 'Beppe yn 'e fleur'. Yn: Ruth, in hân fol ieren. It Hearrenfean, Jongbloed. (Werprinte yn De gielgoerde II).

Kloosterman, Simke 1927. It jubeljier. Snits, Brandenburgh.

Kloosterman, Simke 1936-1944. Ut 'e gielgoerde (I, II). Boalsert, A.J. Osinga.

Kloosterman, Simke 1944. 'Syn libbenswinsk'. Bondele yn: Ut 'e gielgoerde II.

Kloosterman, Simke 1962. Spreuken. Utjûn troch A.I. Brouwer-Prakke. Ljouwert, Miedema. 
Laan, Kornelis ter 1929. Nieuw Groninger woordenboek. Wolters-Noordhoff.

Lennep, Jacob van 1840. De lotgevallen van Ferdinand Huyck. Amsterdam, Meijer Warnars.

Lucassen, Leo \& Lucassen, Jan 2018. Vijf eeuwen migratie. Winnaars en verliezers. Een nuchtere balans van vijfhonderd jaar migratie. Amsterdam, Bert Bakker.

Maupassant, Guy de 1880. 'Boule de Suif'. Bondele yn: E. Zola e.o., Les Soirées de Médan. Parys, Charpentier.

Meertens, P.J. \& Wander, B. 1958. Bibliografie der dialecten van Nederland 1800-1950. Amsterdam, Noord-Hollandsche Uitgevers-Maatschappij.

Meinerts, Eelke 1779. It libben fen Aagtje IJsbrants, of dy Frieske boerinne. Dokkum, H. Groenia.

Meijer, Jaap 1984. Tolk van 't olle volk. Joods supplement op het nieuw Groninger woordenboek. Heemstede. https://www.joodsebibliotheek.nl/ auteur/HEo/Jaap-Meijer/boek/OIo/Tolk-van-t-olle-volk/1/

Meulen, Tsjibbe Gearts 1864. Hirddravery to Flearterp biskrieun fen Mikroskopius en in 't liocht jown fen Waling Dykstra. To Leaauerd, by U. Proost.

Moormann, J.G.M. 2002 (werútjefte fan 1932-4). De Geheimtalen, Het Bargoense standaardwerk, met een nieuw, nagelaten deel. Bezorgd door Nicoline van der Sijs, met een inleiding van Enno Endt.https://www. dbnl.org/tekst/moor012gehe02_01/moor012gehe02_01_0096.php

Nederlandsche zanger 1852. (Anonym:) De Nederlandsche zanger in alle gezelschappen (1852). "Verzameling van meer dan 100 uitgezochte gezelschapsliederen". https://www.dbnl.org/tekst/_ned022nede01_01/ _ned022nede01_01_0004.php

Nijdam, Klaas Cornelis 1828. Botters-goed in mijn liddige aegenblikken (net publisearre).

Noordenbos, Johannes A. 1918. Wiste it noch? Toanielstik yn fjouwer bidriuwen. Berltsum, Westerlaan.

O' Brien, Mary Grantham \& Sarah M. B. Fagan 2016. German Phonetics and Phonology: Theory and Practice. New haven: Yale UP.

Oostendorp, Marc van 2017. 'Nepjiddisch'. Neerlandistiek. Online tijdschrift voor taal- en letterkundig onderzoek, publisearre op 23 febrewaris 2017. https://www.neerlandistiek.nl/2017/02/nepjiddisch/

Oppewal, Teake e.o. (red.) 2006. Zolang de wind van de bomen waait. Geschiedenis van de Friese literatuur. Amsterdam, Bert Bakker. 
Piebenga, J. 1957. Koarte skiednis fan de Fryske skriftekennisse (twadde, útwreide printinge). Drachten, Laverman.

Ploeg, Feike Hiddes van der 1777. Mayke Jakkelis. Sjoch: Brandsma (ed.).

Ploeg, Feike Hiddes van der 1778. Het jonge lieuws boosk. Plesier-spul yn fyf bydrieuwen byschreun. Ljouwert.

Reuter, Fritz 1862-1864. Ut mine Stromtid (Aus meiner Voluntärszeit). https://gutenberg.spiegel.de/buch/ut-mine-stromtid-1383/1

Reuter, Frits (Fritz) [1872]. Gedroogde kruiden. Rotterdam, D. Boll. "Uit het Platduitsch, door G. Velderman".

Riemersma, Trinus 2006. Hoe binne de helten fallen. Bauke de Jong en syn ûnkristlike krústocht. Frjentsjer, útjouwerij Venus.

Sanders, Ewoud 2017a. 'Joods dialect'. Besprek fan Geschiedenis van de joden in Nederland. Ferskynd yn: NRC Handelsblad, 15 juny 2017.

Sanders, Ewoud 2017b. Levi's eerste kerstfeest. Jeugdverhalen over Jodenbekering, 1792-2015. Diss. Nijmegen.

Schuitmaker, Yme Christiaans 1904. Maerteblom. Toanielstik yn fjouwer útkomsten. Ljouwert, R. van der Velde.

Schut, Egbert 1995. De Joodse gemeenschap in de stad Groningen, 16891798. Diss. Grins.

Sijens, Doeke 2001. Sa'n tûzen blauwe skriften. Biografy fan Reinder Brolsma. Boalsert, Koöperative Utjouwerij.

Slofstra, Bouke 2015. 'It wie Babel yn it lyts hjirre'. Ensafh nr. 6 (2015) 4146.

Strauss, Herbert A. \& Hoffmann, Christhard (Hrsg.), Juden und Judentum in der Literatur. Deutscher Taschenbuch Verlag, 1985, 140-171.

Sytstra, Harmen 1841. 'Tsien tuwsen uwt de lottery', yn Tsien tuwsen uwt de lottery, oaf Jouke Rommerts schriften. Frjentsjer, Th. Ypma, 185-191.

Tamminga, Douwe 1961. 'De ynfloed fan it Jiddysk op de Fryske wurdskat'. Taalrubryk 'Taelwarder', Leeuwarder Courant 8-7-1961 (de krant van toen).

TDB: Taaldatabank Fryske Akademy. http://tdb.fryske-akademy.eu/tdb/

Troelstra, Marten Pieters 1869. Nieske Stamsma. En forhael $\hat{u} t \hat{u} s$ tîd. Ljouwert, Jongbloed.

Veen, Hjerre Gjerrits 1856. De kaertlizzer. Ljouwert, Meindertsma Wzn.

Vennemann, Theo 1972. 'Rule Inversion.' Lingua 29, 209-242.

Vries, Abe de 2015. 'Non-konformisme yn de Fryske 'folksskriuwerij'. It Fryske realisme as emansipaasjeliteratuer.' Fers2, nûmer 9, 26 april 2015 (www.fers2.eu). 
Vries, Oebele 1989. 'It skriuwerskip fan Aagtje IJsbrants'. Us Wurk 38, 82103.

Vries, Oebele 2019. 'Hoe't Salomon Levy in ferneamd man wurden is'. De Sneuper nr. 134 (themanummer Salomon Levy), 8-10.

Vries, Oebele \& Breuker, Philippus H. 1987. Fryke almenakstikken út de $18 d e$ en it begjin fan de 19de ieu. Estrik nr 68. Frysk Ynstitút, Grins.

Vriesema, Maaike e.a. 2006. Friesland, wees zacht voor mij. Nieuwkomers in de provincie door de eeuwen heen. Friese Pers Boekerij.

Weg, van der, C.D. 1890. Hwa is yens neiste? Toanielstik mei sang yn fjouwer bidriuwen. Frjentsjer, Telenga.

Weijtens, M.J.P.M. 1971. Nathan en Shylock in de Lage Landen. De Jood in het werk van de Nederlandse letterkundigen uit de negentiende eeuw. Diss. Nijmegen. https://repository.ubn.ru.nl/bitstream/handle/2066/ 147788/mmubn000001_25098864x.pdf

WFT: Wurdboek fan de Fryske Taal / Woordenboek der Friese taal. Ljouwert, Fryske Akademy, 1984-2011.

Winkler, Johan 1874. Algemeen Nederduitsch en Friesch Dialecticon. Diel 1 (1874). Den Haach, Nijhoff. https://www.dbnl.org/tekst/ wink007alge01_01/wink007alge01_01_0033.php

Woude, Siem van der (red.) 2010. Joods leven in Friesland. De Gerdyk, Bornmeer [uitgebreide heruitgave van Woude \& Haan 1995.]

Woude, Siem van der \& Sjoerd de Haan (red.) \& H.G. Joustra (eindred.) 1995. Drie eeuwen Joods leven in Friesland. Ljouwert (Leeuwarden, Stichting Kultuer en Toerisme yn Fryslân.

Zeilstra, R. 1913. Libbensstoarmen. Dronryp, Westerlaan.

Zijlstra, Doeke Hendriks 1889. It testamint. Toanielstik yn 4 útkomsten. Ljouwert, K. van Belkum Kz.

Zondergeld, Gjalt Reinder 1978. De Friese Beweging in het tijdvak der beide wereldoorlogen. Diss. Grins. 